OPEN ACCESS

Edited by:

Zvi Kelman,

National Institute of Standards and Technology, United States

Reviewed by:

James Chong,

University of York, United Kingdom

Michael Trakselis,

Baylor University, United States

*Correspondence:

Robert M. Brosh Jr.

broshr@mail.nih.gov

Specialty section:

This article was submitted to

Molecular Diagnostics and

Therapeutics,

a section of the journa

Frontiers in Molecular Biosciences

Received: 22 January 2018 Accepted: 06 June 2018

Published: 26 June 2018

Citation:

Datta A and Brosh RM Jr (2018) New Insights Into DNA Helicases as

Druggable Targets for Cancer Therapy. Front. Mol. Biosci. 5:59. doi: 10.3389/fmolb.2018.00059

\section{New Insights Into DNA Helicases as Druggable Targets for Cancer Therapy}

\author{
Arindam Datta and Robert M. Brosh Jr.* \\ Laboratory of Molecular Gerontology, National Institute on Aging, National Institutes of Health, NIH Biomedical Research \\ Center, Baltimore, MD, United States
}

Small molecules that deter the functions of DNA damage response machinery are postulated to be useful for enhancing the DNA damaging effects of chemotherapy or ionizing radiation treatments to combat cancer by impairing the proliferative capacity of rapidly dividing cells that accumulate replicative lesions. Chemically induced or genetic synthetic lethality is a promising area in personalized medicine, but it remains to be optimized. A new target in cancer therapy is DNA unwinding enzymes known as helicases. Helicases play critical roles in all aspects of nucleic acid metabolism. We and others have investigated small molecule targeted inhibition of helicase function by compound screens using biochemical and cell-based approaches. Small molecule-induced trapping of DNA helicases may represent a generalized mechanism exemplified by certain topoisomerase and PARP inhibitors that exert poisonous consequences, especially in rapidly dividing cancer cells. Taking the lead from the broader field of DNA repair inhibitors and new information gleaned from structural and biochemical studies of DNA helicases, we predict that an emerging strategy to identify useful helicase-interacting compounds will be structure-based molecular docking interfaced with a computational approach. Potency, specificity, drug resistance, and bioavailability of helicase inhibitor drugs and targeting such compounds to subcellular compartments where the respective helicases operate must be addressed. Beyond cancer therapy, continued and new developments in this area may lead to the discovery of helicase-interacting compounds that chemically rescue clinically relevant helicase missense mutant proteins or activate the catalytic function of wild-type DNA helicases, which may have novel therapeutic application.

Keywords: helicase, DNA repair, replication, genomic instability, small molecule, therapy, cancer

\section{INTRODUCTION}

Targeting the DNA damage response and DNA repair to combat cancer became an attractive hypothesis with the original discoveries made by Thomas Helleday, Alan Ashworth and colleagues that chemicals which inhibit the DNA damage sensor poly(ADP-ribose) (PAR) polymerase 1 (PARP-1) could be used to kill breast cancer cells that are defective in the tumor suppressor genes encoding homologous recombination (HR) repair proteins BRCA1 or BRCA2 (Bryant et al., 2005; Farmer et al., 2005). As elaborated below, there has been much interest in the mechanisms of PARP inhibitors as well as topoisomerase inhibitors used in preclinical and clinical settings, and the 
progress made in these areas have prompted biomedical researchers to investigate these and other potential therapeutic DNA repair proteins as targets to enhance the effects of chemotherapy drugs or ionizing radiation to eradicate cancer cells but spare normal cells, thereby minimizing toxicity usually associated with the DNA damaging treatments. An important aspect of small molecule drugging of at least some DNA repair protein targets involves trapping the enzyme on its DNA substrate resulting in a poisonous protein complex, which will be discussed as a possible precedent for a new class of chemical inhibitors that target DNA helicases, the subject of this review.

DNA helicases are ubiquitous enzymes found in all domains of life and involved in every aspect of nucleic acid metabolism (Crouch and Brosh, 2017). As molecular motors, they utilize the energy derived from binding and hydrolysis of nucleoside triphosphate (typically ATP) to translocate on DNA and disrupt the many hydrogen bonds between complementary strands of the DNA double helix. In addition, certain DNA helicases unwind alternate DNA structures such as triplexes or Gquadruplexes and/or displace proteins bound to single-stranded or double-stranded DNA. DNA helicases play instrumental roles in cellular DNA replication, transcription, DNA repair, and other processes to preserve genomic integrity and maintain cellular homeostasis. Their vital functions are illustrated by the fact that mutations in a number of helicase genes are either linked to hereditary diseases characterized by chromosomal instability or associated with various cancers (Brosh, 2013).

The molecular differences among helicases are of interest as they may provide opportunities for targeting specific helicases in anti-cancer therapies. DNA helicases are broadly categorized according to the grouping (Superfamily (SF)/Family) to which they belong based on sequence homology within conserved motifs in the helicase core domain as well as auxiliary domains residing in the $\mathrm{N}$ - or $\mathrm{C}$-terminal regions of the protein (Umate et al., 2011). Many of the human DNA repair helicases belong to SF2, and the two most prominent families are the RecQ helicases and Iron-Sulfur (Fe-S) helicases which have opposite polarities of single-stranded DNA translocation (Estep and Brosh, 2017). The $3^{\prime}$ to $5^{\prime}$ RecQ helicases (RECQL1, WRN, BLM, RECQL4, RECQL5) share prominent roles in replication fork remodeling, double-strand break (DSB) repair, and regulation of gene expression mediated by their nucleic acid structurespecific binding and catalytic properties as well as their protein interactions. Molecular defects in the RecQ helicases give rise to genomic instability, and mutations in WRN, BLM, and RECQL4 are linked to hereditary diseases characterized by accelerated aging or associated with cancer (Croteau et al., 2014). All five human RecQ helicases are up-regulated in various cancers, suggesting their specialized requirement in rapidly dividing cells to repair replicative lesions or elicit an appropriate response in cell cycle checkpoint or gene expression (Brosh, 2013). As discussed further in the review, structural characterization of various RecQ helicases has provided new insight to the functional importance of key structural elements within the helicase core as well as auxiliary regions that may lead to the design of small molecules which target specific domains.

Given their crucial roles in DNA replication, repair, and genomic stability, DNA metabolic proteins with a characteristic Fe-S cluster have attracted interest from both the basic science and clinical perspectives. Apart from several DNA repair proteins (e.g., DNA glycosylases) and DNA polymerases, certain DNA helicases and helicase-nuclease enzymes possess a conserved Fe-S cluster domain (Wu and Brosh, 2012). The presence of a Fe-S cluster in DNA helicase enzymes was first discovered in XPD, the founding member of a group of DNA repair helicases (DDX11, RTEL-1, FANCJ) that unwind duplex DNA with $5^{\prime}-3^{\prime}$ polarity and are implicated in human chromosomal instability disorders (Rudolf et al., 2006). Research from several labs established that the Fe-S cluster is essential for DNA unwinding by XPD and other Fe-S helicases (Estep and Brosh, 2017), including FANCJ (Wu et al., 2010).

In addition to the SF2 helicases, the SF1 Pif1 helicase is thought to play an important role in nuclear DNA replication (Budd et al., 2006), telomere replication/repair (Geronimo and Zakian, 2016), and mitochondrial DNA synthesis (Lahaye et al., 1991; Pinter et al., 2008). Pif1 may serve to aid the $5^{\prime}$ to $3^{\prime}$ Twinkle hexameric ring-like helicase (SF4) as it generates single-stranded DNA template through difficult-to replicate sequences (Korhonen et al., 2003). For nuclear DNA replication, the ring-like $3^{\prime}$ to $5^{\prime}$ helicase complex constituted by the MCM2-7 proteins (SF6) is essential (Chong et al., 2000). The ring-like structures of the replicative helicases Twinkle (Fernandez-Millan et al., 2015) and MCM complex (Zhai and Tye, 2017), combined with their accessory factors [e.g., mitochondrial single-stranded binding protein (Korhonen et al., 2003), Cdc45/GINS (Aparicio et al., 2009)], enhance the processivity of these helicases to fulfill unwinding of duplexes inherently longer than what is required for strand separation by the helicases implicated in stalled fork remodeling or DNA repair.

In this review, we will provide a framework for thoughtfully considering DNA helicases as a desirable new avenue to target for anti-cancer therapy. In Figure 1, we depict some potential modes of small molecule inhibition of DNA helicases, as well as chemically induced or genetic synthetic lethality that will be referred to in the text. Clearly, an intricate molecular knowledge of helicase conformational states, substrate specificities, protein interactions, pathways, etc. is required to screen for compounds which target helicases successfully in vitro and in vivo with optimal characteristics. We will discuss novel and emerging concepts and developments in anti-cancer therapy as they relate to proposed helicase targets, highly relevant to the prognosis of individuals suffering from many types of cancer that remain a major health risk and source of mortality. Moreover, the current anti-cancer strategies are still highly sub-optimal in many treatments due to the toxicity in normal cells and tissues imposed by chemotherapy drugs and radiation. With the advent of new helicase inhibitors discovered by both high-throughput in vitro assays and in silico compound screening approaches relying on 
A

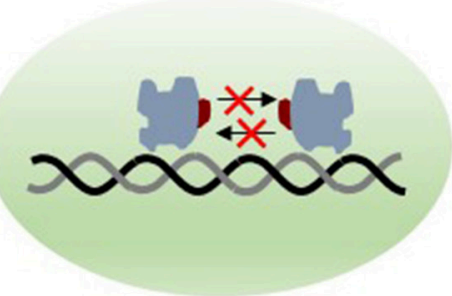

Dimer Interface

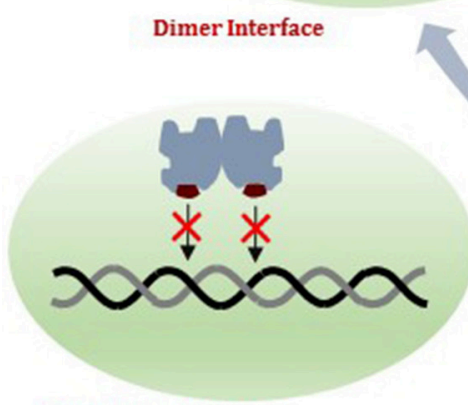

Protein-DNA Interface

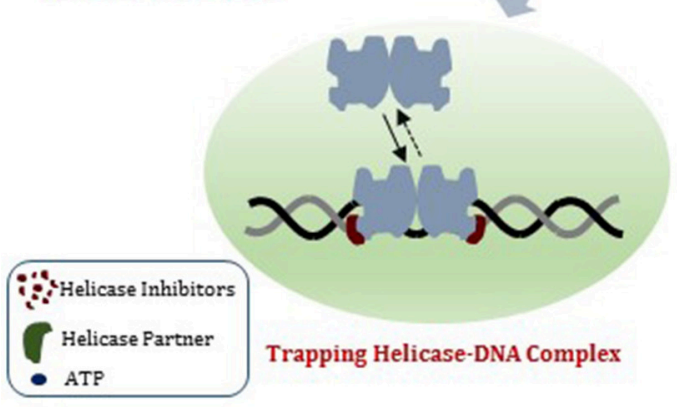

B

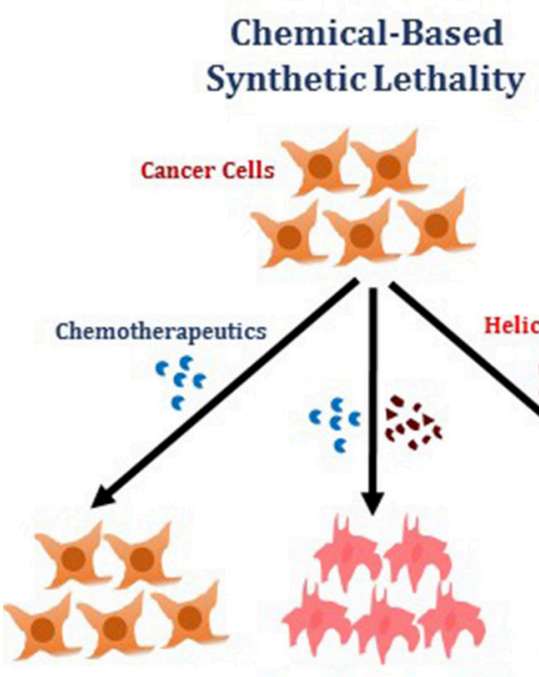

Chemoresistance Synthetic Lethality

\section{Helicase Inhibitors}

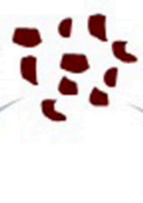

$\longrightarrow$

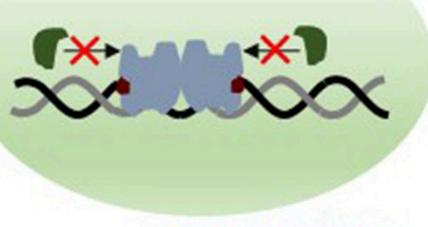

Allosteric Inhibition

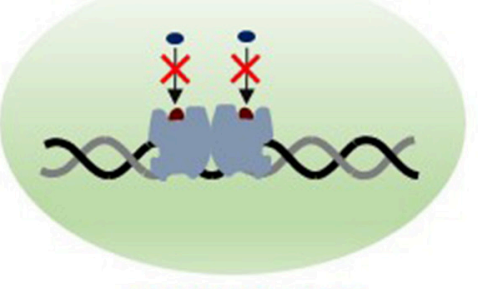

ATP Binding Interface
Orthosteric Inhibition

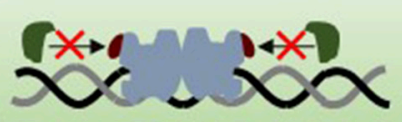


FIGURE 1 | or allosteric mechanisms. Helicase-interacting compounds may also cause the protein to become trapped on DNA, resulting in a toxic complex or lead to the hijacking of other proteins. (B) Two potential strategies for helicase inhibitors (that are not mutually exclusive) are (i) Chemical-based synthetic lethality whereby pharmacological helicase inhibition compromises the cancer cell to chemotherapy DNA damaging drugs or radiation; (ii) Genetic-based synthetic lethality whereby the defined genetic mutant background of the cancer cell is hypersensitive to pharmacological helicase inhibition. See text for details.

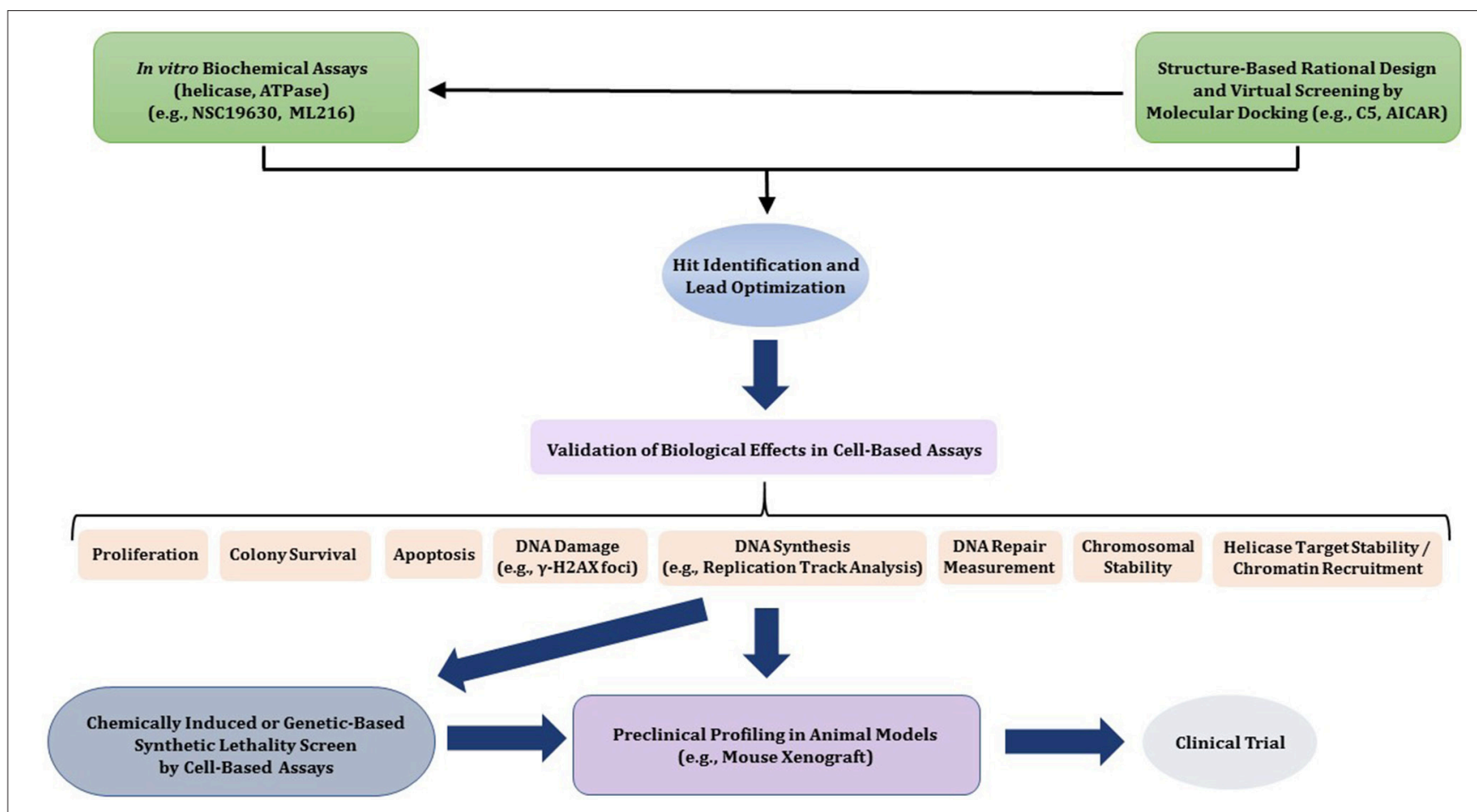

FIGURE 2 | Flow diagram for discovery, optimization, and validation of DNA helicase inhibitors. See text for details.

molecular docking, the stage is set to assess their efficacy using preclinical in vivo models (Figure 2).

\section{DNA DAMAGE RESPONSE PROTEINS: TARGETS FOR CANCER THERAPY?}

The concept of DNA repair or replication stress response modulation for therapeutic intervention has become a hot topic of research and in recent years, clinical pursuit. The field really got its start with the discovery of PARP inhibitors and topoisomerase inhibitors and has taken off with the identification and characterization of novel DNA repair targets. This discussion provides an excellent backdrop for consideration of DNA helicases as potential targets for chemical modulation. From a clinical perspective, personalized medicine has become prominent over the past decade or more. Understanding the genotype-phenotype relationships controlling tumor aggressiveness and their influence over the effectiveness of chemotherapy/radiation treatments has become of increasing importance to the emerging field of DNA damage signaling and DNA repair inhibitors (Velic et al., 2015; Hengel et al., 2017). As illustrated above by the discussion of PARP and topoisomerase inhibitors, their efficacy to combat cancer is dependent on the genetic background of the tumor.

\section{Seminal Discovery of PARP Inhibitors}

Over a decade ago, the concept of DNA repair inhibition emerged in the laboratory setting as a potential avenue for the development of DNA damage response or DNA repair inhibitors with the discovery of small molecules $(<300 \mathrm{Da})$ that deter the molecular and cellular function of PARP (Bryant et al., 2005; Farmer et al., 2005). PARP inhibitors impair the enzyme's ADP-ribose modification function, which in turn suppresses its role in base excision repair, single-strand break repair, and more generally, DNA damage sensing (Cseh et al., 2017). In the case of PARP-1 inhibitors, studies were historically focused on familial and sporadic breast and ovarian cancers with biallelic mutations in the HR repair genes BRCA1, BRCA2, or PALB2 (Hengel et al., 2016; Pommier et al., 2016). The rationale was that pharmacological inhibition of PARP-1 function in rapidly dividing cancer cells would result in an accumulation of single-strand breaks leading to broken replication forks that are essentially DSBs. These DSBs that normally would be repaired by HR with the sister chromatid duplex would be fixed inefficiently in certain cancers due to the absence of any 
one of the three key HR repair proteins BRCA1, BRCA2, or PALB2. Although some success was achieved for treatment of ovarian cancer patients with the PARP-1 inhibitor olaparib, resistance to the drug has limited its therapeutic effectiveness (Murata et al., 2016), leaving researchers to continue exploring and developing new and better cancer therapies focused on PARP inhibitors targeting each member of the family and optimal cotreatment strategies of these compounds with other anti-cancer agents.

\section{Development of Clinically Relevant Topoisomerase Inhibitors}

Topoisomerase inhibitors are perhaps as high profile as the PARP inhibitors for potential clinical use (Pommier et al., 2010). The discovery and development of topoisomerase inhibitors that cause cytotoxicity in cancer cells has sparked tremendous interest in their suitability for antibacterial and anti-cancer applications. Much has been learned about the mechanism of action of topoisomerasepoisoning inhibitors, and it is postulated that many of these compounds act in a similar manner to certain PARP inhibitors by trapping the enzyme on DNA (see below).

Researchers are engaged in the quest to discover more effective topoisomerase (as well as PARP) inhibitors that can hit every cellular target, are chemically stable and behave optimally according to pharmacokinetic parameters. In addition, chemotherapy drug combinations need to be optimized. Aside from these challenges, the looming concerns for compounds that impair the functions of other DNA repair proteins is their effective targeted drug delivery and sub-optimal therapeutic index (Hengel et al., 2017). Further studies that elucidate the pathways whereby such inhibitors act in cells to exert their cytotoxicity and optimize tumorspecific delivery approaches are high priorities in the field. Moreover, combination therapies that exploit the genomic signature of a tumor may lead to the development of anti-cancer strategies which lower the cancer-killing drug doses, thereby sparing normal cells and tissues. Such efforts in precision medicine have become paramount ( $\mathrm{O}^{\prime} \mathrm{Connor}$, 2015).

\section{Trapping PARP- and Topoisomerase-DNA Complexes Presents a Paradigm for New Anticancer Drugs}

Foreshadowing the potential mechanism of action of DNA helicase inhibitors (discussed below), research has revealed that some chemical PARP inhibitors and topoisomerase inhibitors act by trapping the enzyme on DNA, thereby poisoning cells via the formation of toxic DNA-proteindrug complexes that have consequences beyond simply inhibiting catalytic function (Pommier et al., 2015). Yves Pommier and colleagues first used the term interfacial inhibitors to describe drugs that trap protein-DNA complexes by binding at their interfaces, and the concept has been expanded to include medicinal compounds that bind at protein-protein interfaces as well (Pommier and Cherfils, 2005). An excellent example of the former, highly germane to certain emerging DNA repair inhibitors, is represented by those compounds which inhibit topoisomerase action by binding to the very site where the enzyme interacts with DNA to cleave its phosphate backbone, resulting in a trapped topoisomerase inhibitor-stabilized cleavage complex (Pommier, 2013).

In parallel to such interfacial topoisomerase inhibitors, certain PARP-1 and PARP-2 inhibitors act by trapping PARP on DNA in vitro, helping to explain why cellular exposure to these PARP-binding drugs exerts a greater cytotoxicity than the absence of PARP altogether (Pommier et al., 2016). The development of PARP trapping assays using extracts of PARP inhibitor-treated cells to assess chromatin enrichment or employing purified PARP recombinant proteins and fluorescently or radioactively labeled oligonucleotide-based DNA substrates with site-specific damage (e.g., single-strand nick) incubated with PARP inhibitor has provided seminal evidence for DNA-bound PARP complexes. From a more clinical perspective, there is great interest in understanding the mechanisms for resistance to PARP inhibitors such as PARP expression, drug efflux, and changes in DNA damage response/DNA repair in response to PARP inhibitor exposure. In addition, ongoing efforts in anti-cancer therapy focus on developing successful combinations of PARP inhibitor with other DNA damaging treatments and/or DNA repair inhibitors. Studies of the interfacial PARP inhibitors and topoisomerase inhibitors will likely serve as models for the future investigations of DNA helicase inhibitors that behave according to a similar enzyme trapping mechanism; however, these mechanistic analyses are only in their infancy (see below).

\section{New Targets in Genomic DNA Metabolism to Enhance Cancer Therapy}

While topoisomerase and PARP inhibitors continue to attract interest as targets for anti-cancer therapy, in recent years other DNA damage response and DNA repair targets have emerged. For the small molecules that inhibit these proteins, some of the same basic idealized principles apply in which the chemical agents acting as a monotherapy or in combination with other chemotherapy treatments will target the cancerous cells and tissues, sparing normal ones by exploiting a therapeutic threshold index. Some examples of new DNA repair inhibitors that target HR repair proteins (e.g., RAD51, RAD52, RAD54), the structure-specific nuclease MRE11, and others are summarized in recent reviews (Huang and Mazin, 2014; Velic et al., 2015; Hengel et al., 2017). These relatively new targets, like the PARP- and topoisomerase-interacting drugs, may provide insights to mechanistic aspects of helicase inhibitors and their application in anti-cancer regimes. For example, small molecule inhibitor-induced trapping of DNA metabolic proteins may represent a more generalized mechanism with poisonous consequences applicable to compounds that target other DNA repair proteins such as DNA methyltransferases and DNA helicases. 


\section{DNA REPAIR INHIBITORS IDENTIFIED BY HIGH-THROUGHPUT SCREENS AND MOLECULAR DOCKING APPROACHES}

As mentioned above, small molecule inhibitors of proteins implicated in DNA damage signaling and DNA repair identified by high-throughput screens (HTS) have been advanced by basic research efforts in part to develop anti-cancer strategies to enhance chemotherapy or radiation treatments. These assays can be broadly divided into two categories: (1) Biochemical screens which are used to directly assess modulation of protein function, be it enzymatic activity or ligand binding; (2) Cellbased assays used to investigate if a set of compounds influence a DNA repair pathway, ultimately with an outcome on cellular homeostasis and/or genomic stability. Often, once a compound is identified that is potent and specific for its target in vitro, chemists will optimize its structural and solubility properties for in vivo application.

In the following sections, we will highlight some examples of DNA repair inhibitors and DNA helicase inhibitors discovered by HTS and molecular docking approaches. Practically all the presently known helicase inhibitors have been shown to act synergistically in a genetic or chemical manner with druggable DNA repair targets in cell-based systems, and some of these synergistic combinations will be discussed.

\section{Structure-Based Design of DNA Repair Inhibitors by Molecular Docking Approaches}

Although significant advancement has been made in developing potential small molecule inhibitors targeting DNA repair machinery, only a few have reached the clinic so far. Hits primarily identified by HTS based on in vitro biochemical assays sometimes fail to exert their desired effect at the cellular level and are often non-specific. In general, the associated cost, time, assay complexities and screening quality are considered as major challenges in developing highly potent and specific drug-like molecules using experimental HTS approaches (Shoichet et al., 2002; Moitessier et al., 2008; Awate and Brosh, 2017). With the rapid advancement in computational methodologies coupled with the availability of high-resolution crystal structures of target proteins, structurebased virtual screening of large compound libraries has drawn significant attention in modern drug discovery research over the last two decades. The approach has been successfully used to identify highly accurate lead molecules in a timeand cost-effective manner (Kroemer, 2007; Meng et al., 2011). Among the various structure-based in silico compound screening methodologies, molecular docking technique is widely adopted and considered as the principal one. Given the three-dimensional structure of the target protein, this important computational tool allows the researchers to virtually screen a large set of small organic molecules and provides information about the binding mode and strength of the binding for individual protein-ligand complexes. Therefore, molecular docking is useful not only in identifying new hits but also in facilitating the further optimization of the pre-identified lead molecules to develop more potent analogs.

\section{SMALL MOLECULE INHIBITORS OF DNA HELICASES}

Although somewhat lagging behind in the field of small molecule DNA repair inhibitors, pharmacological inhibition of DNA helicases has begun to attract interest. A recent review summarizes experimental approaches to identify and characterize DNA helicase inhibitors by biochemical and cellbased assays (Banerjee et al., 2016). With the discovery of new helicase protein structures and a growing understanding of their molecular mechanisms, there has been increasing interest in small molecules that modulate helicase function. Below, we provide the reader a current assessment of the field. Given the number of both DNA and RNA helicases implicated in fundamentally important areas of nucleic acid metabolism in human cells, it seems likely that continued advances in pharmacological interventions will be made. These advances should provide unique tools to investigate the cellular functions of helicases, their biological pathways in nucleic acid transactions, and further development in pre-clinical models (Figure 2).

While some helicase inhibitor studies have focused on pharmacological modulation of human DNA helicases involved in DNA damage responses that would affect the efficacy of ionizing radiation or chemotherapy treatments, a number of inhibitors of viral helicases have been discovered over the past decade that may be useful for suppressing viral diseases (Shadrick et al., 2013). The clinical success of a herpes simplex virus (HSV) helicase-primase complex inhibitor known as Amenamevir (ASP2151) is a strong testament to viral helicases as potential druggable targets to deter viral pathogenesis (Chono et al., 2010; Katsumata et al., 2012; Tyring et al., 2012). ASP2151 has a broad anti-herpes virus spectrum including HSV-1, HSV-2, and varicella zoster virus as well as acyclovirresistant thymidine kinase-deficient HSV strains (Chono et al., 2010; Himaki et al., 2012). Although not yet clinically proven, small molecule-based approaches showed significant progress in targeting other viral helicases such as human papilloma virus (HPV) and hepatitis C virus (HCV) helicases. By highthroughput screening and subsequent chemical optimization, a family of biphenylsulfonacetic acid-based small molecules was discovered that inhibit the ATPase and helicase activity of HPV6 E1 helicase in vitro, but their bioactivity remains elusive (Faucher et al., 2004). On the other hand, a novel class of small molecules that specifically antagonizes the physical interaction of HPV E1 helicase with E2 protein was found to be effective to inhibit HPV DNA replication in cell-based assays (White et al., 2003; Yoakim et al., 2003). This work serves as an excellent example of targeting viral helicase protein-protein interactions to develop potential antiviral therapies. As reviewed elsewhere, the hepatitis C virus NS3 helicase is considered a potential candidate for specifically targeted antiviral therapy (Belon and 
Frick, 2009). Lead compounds (e.g., Soluble blue HT, Acridone4-carboxylic acid derivatives, benzotriazoles) that inhibit HCV helicase activity and impair cellular HCV RNA replication hold immense potential. For a comprehensive review of new and developing antiviral and antibiotic small molecule inhibitors, see (Shadrick et al., 2013).

\section{In Vitro and Cell-Based Properties of Known Compounds That Inhibit DNA Unwinding Catalyzed by Human Helicases}

Table 1 lists recently identified small molecular inhibitors of human DNA helicases. Most of the small molecule helicase inhibitors were identified from in vitro helicase assays using purified recombinant DNA helicase proteins, oligonucleotidebased DNA substrates, and small molecule libraries. However, a virtual screen of FDA-approved drugs by a nuclease-based assay identified an inhibitory compound for the DNA2 helicasenuclease implicated in Okazaki fragment processing during DNA replication (Liu et al., 2016). Of those tested in cell-based assays, small molecule inhibitors of DNA unwinding catalyzed by WRN (Aggarwal et al., 2011, 2013b), BLM (Nguyen et al., 2013), and DNA2 (Liu et al., 2016; Kumar et al., 2017) all negatively affect proliferation of cancer cells and induce DNA damage and/or chromosomal instability. Moreover, these helicase inhibitors behave synergistically with other compounds that induce DNA damage, inhibit DNA repair, or impose replication stress. Certain helicase inhibitors operate in a manner that is dependent on the presence of the DNA helicase target (Aggarwal et al., 2011, 2013b; Nguyen et al., 2013; Liu et al., 2016), suggesting that pharmacological inactivation of helicase function involves the interference of a genome maintenance pathway which is distinct from the effect imposed by the absence of the helicase altogether. Presumably, backup mechanisms are elicited in certain helicasedeficient backgrounds, whereas a helicase inhibitor complex with its target imposes uniquely deleterious effects, akin to those caused by a protein trapping mechanism discussed earlier. While an inhibitor of the RECQL1 helicase was identified from an in vitro helicase activity screen (https://pubchem.ncbi.nlm.nih. gov/bioassay/2708), no published advances in terms of effects of the small molecule on functionality or metabolism of human cells have been reported. In the following sections, we will discuss some key features of the human DNA helicase inhibitor studies to provide the reader a sense of the field's current advances and future directions with an eye on clinical applications.

\section{WRN}

Bi-allelic mutations in the WRN gene result in Werner syndrome (WS), a progeroid disease considered by many to most closely resemble accelerated aging (Oshima et al., 2017). With the apparent exception of neurodegeneration, almost all clinical features associated with normal aging (e.g., heart disease, osteoporosis, diabetes, cataracts, etc.) are observed early in life (concomitant with the adolescent growth spurt) for those individuals diagnosed with WS. Although the WRN gene product has been studied by molecular biologists, biochemists, cell biologists, and clinicians for over 2 decades, it is still unclear the molecular defects of WRN that are responsible for the mutant cellular phenotypes which include chromosomal instability, replication and DNA damage response defects, and abnormal transcriptional regulation. The WRN gene encodes a protein that has both DNA helicase activity and DNA exonuclease activity. Moreover, WRN interacts with a large cast of proteins implicated in various DNA transactions important for DNA repair, the replicational stress response, and telomere capping, suggesting that it may have pleiotropic roles. Although WRN mutant mouse models, particularly those crossed with other mutant mice, have provided some clues to WRN's involvement in telomere metabolism (Chang et al., 2004; Du et al., 2004; Laud et al., 2005), the definitive molecular and cellular deficiencies underlying WS remain elusive.

To provide a fresh approach to studying WS, we conducted a search for small molecules that behaved as potent and specific WRN helicase inhibitors (Aggarwal et al., 2011). The in vitro-based compound screen using a conventional biochemical DNA unwinding assay was performed to identify inhibitors of purified recombinant human WRN helicasecatalyzed DNA unwinding and positive hits were tested in cell-based assays. These efforts led to the identification and characterization of the compound NSC 19630 from the National Cancer Institute (NCI) Diversity Set which inhibited WRN-catalyzed DNA unwinding of a forked duplex DNA substrate $\left(\mathrm{IC}_{50} \sim 20 \mu \mathrm{M}\right)$ in a specific manner based on the observation that other purified recombinant DNA helicase proteins tested (e.g., BLM, RECQL1) were either not inhibited or only modestly affected at much greater compound concentrations (Table 1). The inhibitory effect of NSC 19630 on WRN DNA unwinding was not mirrored by a similar effect on DNA binding or ATP hydrolysis, suggesting that the compound specifically interfered with WRN's strand separation activity. Biological studies with NSC 19630 and the human cervical cell line HeLa demonstrated that the WRN helicase inhibitor negatively affected cell proliferation and replication, as well as induced DNA damage and apoptosis in a WRNdependent manner based on the observation that the same cells depleted of WRN by RNA interference were resistant to the effects of NSC 19630. Further studies showed that NSC 19630 behaved synergistically with the topoisomerase inhibitor topotecan, the PARP1 inhibitor KU0058948, or the G-quadruplex binding ligand Telomestatin for inhibiting proliferation and inducing DNA damage (Aggarwal et al., 2011), suggesting that pharmacological inhibition of WRN helicase activity under conditions of replicational stress or DNA damage severely compromised the cellular response.

In subsequent work, a compound designated NSC 617145 that is structurally related to NSC 19630 was found to inhibit WRN helicase activity with even greater potency $\left(\mathrm{IC}_{50} \sim\right.$ $230 \mathrm{nM}$ ) and render human cells deficient in the Fanconi Anemia (FA) pathway hypersensitive to the DNA cross-linking agent mitomycin C (MMC) in a WRN-dependent manner (Aggarwal et al., 2013b) (Table 1). Interestingly, NSC 617145 did not sensitize FA-deficient cells to hydroxyurea (HU) (which causes replication stress by depleting the deoxynucleotide phosphate pool), suggesting that DNA DSBs that accumulate when the FA 
TABLE 1 | Small molecule inhibitors of human DNA helicases.

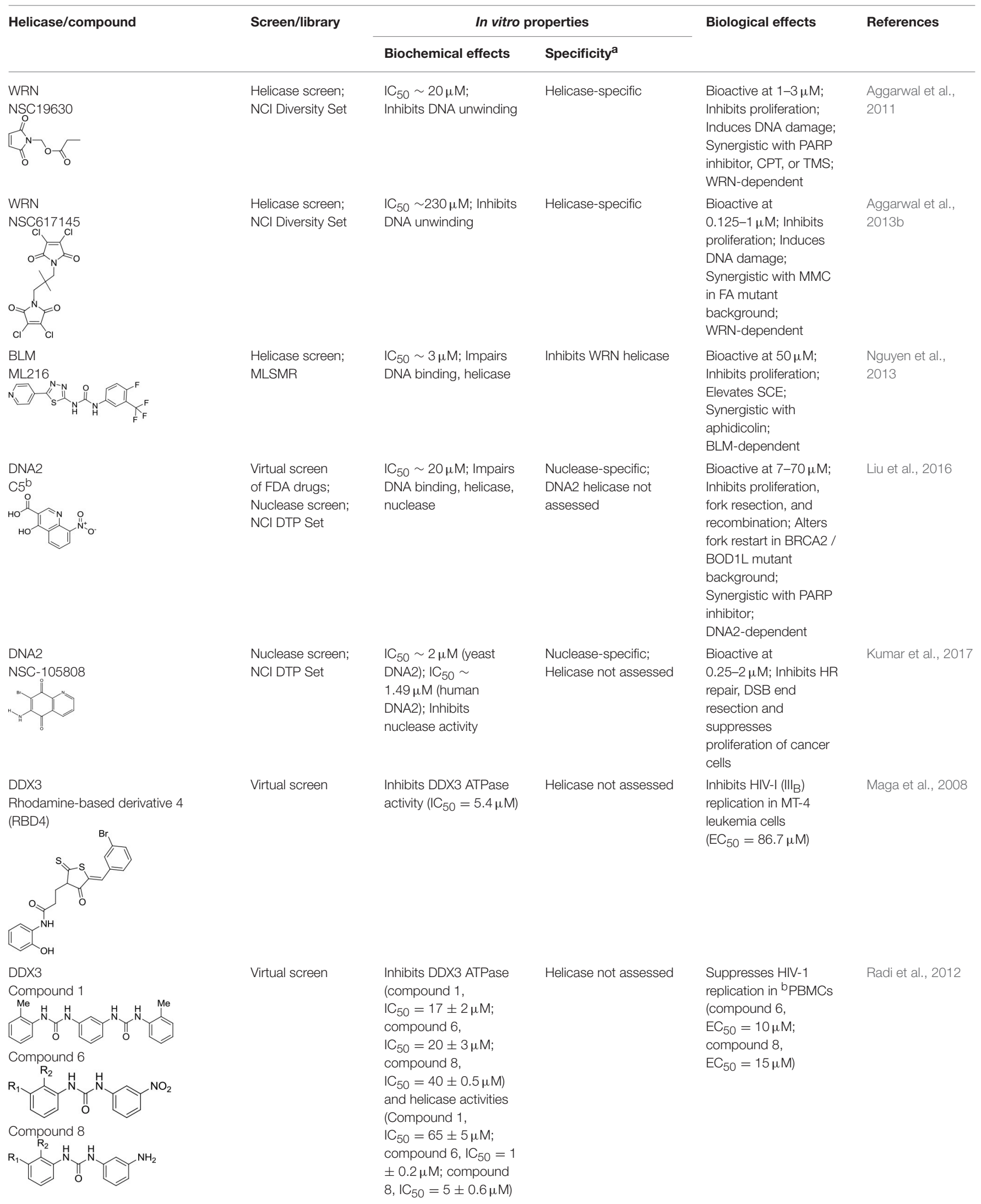


TABLE 1 | Continued

\begin{tabular}{|c|c|c|c|c|c|}
\hline Helicase/compound & Screen/library & \multicolumn{2}{|c|}{ In vitro properties } & Biological effects & References \\
\hline $\begin{array}{l}\text { Mcm2-7 } \\
\text { Ciprofloxacin }\end{array}$ & Helicase screen & $\begin{array}{l}\mathrm{IC}_{50} \sim 632 \mu \mathrm{M} \text {; Inhibits } \\
\text { DNA } \\
\text { unwinding }\end{array}$ & Helicase-specific & $\begin{array}{l}\text { Bioactive at } \\
520-670 \mu \mathrm{M} \text { (yeast) } \\
\text { and } 160-350 \mu \mathrm{M} \\
\text { (human cells); Inhibits } \\
\text { proliferation of yeast } \\
\text { and human cells }\end{array}$ & Simon et al., 2013 \\
\hline
\end{tabular}

a Specificity determined by its effect on other DNA helicases.

${ }^{b}$ DNA2 nuclease, but not helicase activity, was assessed.

pathway is crippled in its ability to respond to interstrand crosslinks (ICL) are particularly problematic in the face of poor WRN helicase activity as opposed to simply stalled forks. Perhaps WRN may aid in the repair of such DSBs by mediating HR. Supporting the hypothesis that the interaction of the helicase inhibitor with WRN causes the formation of a toxic ternary complex with genomic DNA that interferes with normal DNA repair, NSC 617145 treatment enriched WRN in the chromatin fraction of human cells (Aggarwal et al., 2013b). It remains to be seen if (or what) other DNA replication/repair factors are sequestered with WRN in the chromatin fraction due to the presence of the drug. This is particularly relevant as protein hijacking may contribute to the cytotoxicity of the WRN helicase inhibitor.

Further studies with NSC 617145 revealed that the drug is even more toxic in human cells that are doubly deficient in the FA pathway and DNA Protein Kinase $\mathrm{C}\left(\mathrm{PK}_{\mathrm{cs}}\right)$, a DNA damage sensor and phosphorylating enzyme which is implicated in non-homologous end-joining (NHEJ) pathway of DSB repair (Aggarwal et al., 2013a). This finding suggests that the toxicity imposed by WRN helicase inhibition in the context of ICL-induced DNA damage does not derive from a self-imposed deleterious NHEJ pathway. Moreover, the results provide further evidence that WRN helicase inhibitors can induce synthetic lethality via a genetic-based and/or chemically induced mechanism. These results, along with those from other studies in the DNA damage response/DNA repair field, continue to spark interest in the development of anti-cancer strategies that exploit the genetic background of a tumor as well as drug combinations that together might overwhelm that resistance of tumors to mono-therapies. Moving toward a pre-clinical application is a priority in the DNA repair community, which will require advancement through model genetic systems and in vivo applications.

It seems likely that small molecule helicase inhibitors like those directed against WRN will operate in a manner that is dependent on the genetic background of the tumor. WRN, like other human RecQ helicases, is typically up-regulated in its expression in various cancer cell lines; moreover, their down-regulation by RNA interference has been shown to cause decreased proliferation (Brosh, 2013). Therefore, inhibition of WRN function may represent a useful strategy to compromise rapidly dividing cancer cells dependent on WRN to deal with replicative lesions. Analysis of the NCI 60 cancer cell database did not show a strong correlation between WRN protein level and sensitivity to NCS 19630 (Aggarwal et al., 2011), indicating a more complex scenario. Nonetheless, non-cancerous breast epithelial cells or normal fibroblasts were found to be resistant to NSC 19630 (Aggarwal et al., 2011), suggesting that a therapeutic threshold for the WRN inhibitor may come into play. Interestingly, it was reported that the susceptibility of breast cancer cells to killing by camptothecin (CPT) correlated with CPT-induced WRN degradation (Shamanna et al., 2016). Exposure to the WRN helicase inhibitor NSC 617145 was also observed to cause WRN degradation (Aggarwal et al., 2013b), suggesting that the anti-proliferative effects of compounds that target WRN or protein partners with which it interacts (e.g., topoisomerase I) is a causative factor. Further studies in this area may help to elucidate strategies to target tumors by exploiting their genetic background and negatively affecting the activity as well as the stability of DNA repair protein targets with small molecules.

\section{BLM}

Bi-allelic mutations in the BLM gene result in Bloom's syndrome (BS) characterized by a pronounced predisposition to all types of cancer and certain features of accelerated aging (de Renty and Ellis, 2017). A prominent form of chromosomal instability used to clinically diagnose BS is elevated sister chromatid exchange (SCE) that is attributed to defects in recombinational repair and a poor replication stress response. The BS helicase (BLM) shares sequence homology within the conserved ATPase/helicase core domain of WRN and RecQ orthologs. In addition, BLM (as well as WRN) contains a conserved RecQ C-terminal (RQC) region that bears $\mathrm{Zn}^{2+}$-binding and winged helix $(\mathrm{WH})$ domains and a 
Helicase RNase D-like C-terminal (HRDC) domain (see below). The RQC is implicated in structure-specific DNA binding and protein interactions of WRN and BLM (Estep and Brosh, 2017), and the HRDC is implicated in ligand-induced conformational changes in BLM (Newman et al., 2015).

To gain greater insight to BLM's molecular and cellular roles in DNA metabolism, a HTS of greater than 350,000 compounds from the Molecular Libraries Small Molecule Repository (MLSMR) was undertaken to identify inhibitors of BLM-catalyzed helicase activity on a fluorometric-labeled forked duplex DNA substrate (Nguyen et al., 2013). For this assay, a recombinant truncated version of BLM containing the helicase core domain but lacking 119 amino acids in the Nterminus and 635 residues in the $\mathrm{C}$-terminus was used. Of the positive hits for BLM helicase inhibition, one compound was optimized for its medicinal chemistry properties (e.g., solubility, cell permeability), leading to ML216 (Table 1) as a lead candidate for further studies. The $\mathrm{IC}_{50}$ for ML216 inhibition of helicase activity catalyzed by the BLM helicase domain fragment or full-length BLM was determined to be in the low micromolar range. Interestingly, ML216 was much less effective in inhibiting branch-migration of synthetic Holliday Junction (HJ) or mobile D-loops DNA substrates, as well as a G-quadruplex DNA substrate, with an $\mathrm{IC}_{50}$ value of $\sim 50 \mu \mathrm{M}$ (Nguyen et al., 2013). The differential effect of ML216 on BLM catalytic activity with these different DNA substrates would suggest that the compound affects BLM in a unique manner as it unwinds forked duplex DNA, and that BLM operates by distinct DNA structure-specific mechanisms during DNA unwinding and branch-migration, as suggested by previous studies (Mazina et al., 2012). Although ML216 displayed specificity for helicase inhibition based on results from assays with UvrD, RECQL1, and RECQL5 helicases $\left(\mathrm{IC}_{50}>50 \mu \mathrm{M}\right)$, the small molecule inhibited forked duplex DNA unwinding by a WRN helicase domain fragment or fulllength WRN at significantly lower drug concentrations (Nguyen et al., 2013). The $\mathrm{IC}_{50}$ value for inhibition of forked duplex DNA unwinding by full-length WRN was only 1.7-fold greater than $\mathrm{IC}_{50}$ value for inhibition of unwinding by full-length BLM on the same partial duplex DNA substrate. This raises the possibility that ML216 binds to both BLM and WRN through their conserved ATPase/helicase core, RQC, or HRDC domains; however, biophysical mapping studies are required to address this. ML216 was shown to inhibit BLM binding to singlestranded DNA or forked duplex DNA (Nguyen et al., 2013), suggesting that the compound inhibits BLM-catalyzed DNA unwinding by interfering with its DNA binding function, but an analysis of ML216's effect on WRN DNA binding was not reported in the study.

A structurally related analog of ML216 (5-(pyridin-4-yl)1,3,4-thiadiazol-2-amine derivative, designated as compound 33) was achieved through medicinal chemistry efforts focused on structure-activity relationships (SAR) (Rosenthal et al., 2013). Like ML216 (Nguyen et al., 2013), compound 33 inhibited BLM helicase activity and single-stranded DNA binding, consistent with its non-ATP competitive inhibition of DNA-dependent BLM ATPase activity (Rosenthal et al., 2013). Compound 33 showed a greater selectivity for inhibition of BLM helicase activity over WRN compared to ML216. Although compound 33 also inhibited single-stranded DNA binding by WRN, its effect was not quite as potent as that observed for BLM DNA binding.

From a preclinical perspective, an initial litmus test for the medicinal development of a small molecule DNA repair inhibitor is its activity in cell-based models. ML216 was observed to inhibit proliferation of human SV40-transformed skin fibroblasts in a BLM-specific manner, i.e., the presence of BLM in the isogenic cell line was required for ML216 $(12.5$ or $50 \mu \mathrm{M})$ to inhibit proliferation in the 48 or 72-h time-period (Nguyen et al., 2013). Because elevated SCE is such a pronounced phenotype of BS, the effect of BLM small inhibitors ML216 (Nguyen et al., 2013) and compound 33 (Rosenthal et al., 2013) on this form of chromosomal instability in human cells was assessed. Both BLM inhibitors induced SCE in BLM-positive, but not BLMnegative cells, consistent with a BLM-dependent effect. Preexposure to $50 \mu \mathrm{M}$ ML216 for 24-h sensitized human cells to the DNA polymerase inhibitor aphidicolin in a BLM-dependent manner (Nguyen et al., 2013). A 48-h pre-exposure to ML216 caused a greater frequency of $\gamma$-H2AX foci (a marker of DSBs) induced by MMC in a BLM-dependent manner as well. Thus, the results suggest that the BLM helicase inhibitor ML216 as well as compound 33, like the WRN helicase inhibitors NSC 19630 and NSC 617145, behave in a dominant-negative fashion, relying on their helicase target to cause anti-proliferative and DNA damage-inducing effects. However, both ML216 (Nguyen et al., 2013) and compound 33 (Rosenthal et al., 2013) impaired DNA binding by BLM [whereas, the WRN inhibitor NSC 19630 did not appreciably affect WRN DNA binding at drug concentrations in which significant helicase inhibition was observed (Aggarwal et al., 2013b)], suggesting that the BLM inhibitors may not trap BLM helicase protein on genomic DNA in cells. This is contrasted to NSC 617145 , which was reported to enrich WRN's association with chromatin (Aggarwal et al., 2013b).

\section{DNA2}

DNA2 is a protein with dual helicase and endo-/exo-nuclease activities originally discovered in yeast to play an important role in processing of DNA replication intermediates (Budd et al., 1995, 2000). These advancements laid the foundation for studies of DNA2 in human cells, which also revealed its importance in DNA metabolism. Emerging evidence indicates that in addition to DNA2's involvement in Okazaki fragment processing (Kang et al., 2010b), the helicase-nuclease is important for DNA endprocessing as an early step in DSB repair (Symington, 2016) and nucleolytic processing of stalled or regressed forks that arise during replication stress (Thangavel et al., 2015). These findings, coupled with observations that DNA2 is overexpressed in various cancers, has made DNA2 an attractive candidate for inhibition as a strategy for cancer therapy (Jia et al., 2017). Indeed, several groups have reported that DNA2 depletion by RNA interference causes the reduced proliferation of cancer cells (Jia et al., 2017). Thus, DNA2 may be suitable for chemical inhibition by small molecules that inhibit its catalytic nuclease and/or helicase function.

A HTS with a fluorometric DNA substrate and yeast DNA2 was employed to search for inhibitors of the enzyme's nuclease 
activity (Kumar et al., 2017). From this screen of $\sim 50,000$ compounds, a couple of compounds (NSC 5195242, NSC 105808) were identified that could inhibit yeast and human DNA2 nuclease in a specific manner (by its negligible effect on other nucleases tested) (Table 1). However, these compounds were not tested on nuclease-dead versions of DNA2 to assess if they affected DNA2 helicase activity. It was found that NSC 105808 did not affect DNA2 ATPase (Kumar et al., 2017). NSC 105808 negatively affected proliferation of human bone osteosarcoma U2OS cells, and its anti-proliferative effect was suppressed by ectopic expression of DNA2 at a level 1.5-2.0-fold greater than endogenous DNA2, leaving the authors to propose that DNA2 is the target of the compound (Kumar et al., 2017). However, NSC 105808 was not tested on DNA2-deficient or DNA2-depleted cells, so a comparison to the mechanisms of action for the reported WRN- or BLM-specific helicase inhibitors which impaired cell proliferation in a manner that was dependent on the presence of either RecQ helicase in human cells has not been done. Nonetheless, it may be speculated that a small molecule which causes the trapping of a helicase on the DNA, such as WRN inhibitor NSC 617145 (Aggarwal et al., 2013b), may behave quite differently from a DNA2 nuclease inhibitor such as NSC 105808. Further studies are warranted to characterize the cell-based effects of chemical DNA2 nuclease inhibitors.

Both DNA2 nuclease inhibitors NSC 105808 and NSC 5195242 inhibited DNA end processing in a reconstituted system (Kumar et al., 2017). Moreover, NSC 105808 was observed to diminish DNA end-resection and HR in human cells. In addition, NSC 105808 suppressed the sensitivity of FANCD2 -/- cells to cisplatin, like the effect of DNA2 depletion (Kumar et al., 2017), suggesting that the compound targets DNA2. In several different cancer cell models, the DNA2 inhibitor suppressed proliferation of cancer cells with oncogene-induced replication stress (Kumar et al., 2017), suggesting a potential avenue of further exploration for cancer therapy. Studies with DNA2 inhibitors applied to genetic organisms and mouse xenografts that serve as good preclinical models will help to address the usefulness of these compounds for further development.

\section{Mcm2-7}

The mini-chromosome maintenance protein 2-7 (Mcm2-7) is a well conserved hexameric DNA helicase that plays an essential role in DNA replication by unwinding the parental duplex strands to be copied (Abid Ali and Costa, 2016). The observations that certain mutations in the $\mathrm{Mcm}$ helicase subunits are associated with cancer and that $\mathrm{Mcm}$ is over-expressed in cancer cells supports the idea that this hexameric helicase complex is a suitable target for cancer therapy (Neves and Kwok, 2017). Currently, only very limited work has been done to identify inhibitors of DNA unwinding by $\mathrm{Mcm}$ complexes. It was determined that helicase activity catalyzed by the Mcm467 subcomplex was inhibited by heliquinomycin (Ishimi et al., 2009); furthermore, this compound decreased proliferation of cancer cells grown in culture (Toyokawa et al., 2011) (Table 1). More recent efforts in this area led to the identification of a fluoroquinoline antibiotic known as ciprofloxacin (previously shown to deter the catalytic function of topoisomerase II) as an inhibitor of the Mcm2-7 helicase (Simon et al., 2013) (Table 1). Ciprofloxacin was shown to inhibit the growth of yeast cultures, and one of the mcm mutant strains tested was resistant to the compound, suggesting that $\mathrm{Mcm} 2-7$ is a target of the drug.

\section{SUCCESSFUL VIRTUAL SCREENS TO DISCOVER HELICASE INHIBITORS}

We expect these seminal studies to be followed by new compound inhibitors designed by molecular docking with recently solved DNA helicase structures. Most of the docking programs perform two basic operations, "docking" and "scoring." Ligands are docked into the protein structure to predict most possible conformations of the protein-ligand complexes, particularly the conformations of the ligands bound to the binding pockets of the target protein. In the second operation, using a scoring function, the binding affinities of the individual ligands to the target protein in each conformational state are calculated and thus multiple ligands are ranked according to their respective docking score. In the following sections, we will discuss advances to identify helicase inhibitors by virtual screening.

\section{DDX3}

Resistance of HIV-1 to the commonly used anti-HIV drugs is often due to drug-induced acquired mutations in the viral enzymes. Targeting host cell cofactors holds immense therapeutic potential because they are less susceptible to drug-induced mutability compared to the viral enzymes (Kwong et al., 2005). Cellular RNA helicases (e.g., RNA Helicase A (RHA), RNA Helicase 116 (RH116), DEAD-box helicases DDX1 and DDX3) play crucial roles in HIV-1 replication inside the host cells and may represent good targets, provided that cytotoxicity is not a factor. Cell-based screening of a series of ring-expanded nucleoside (REN) analogs identified a potent small molecule inhibitor (CID 44586781) of DDX3, an ATP-dependent RNA helicase required for exporting HIV-1 RNA from the nucleus to cytoplasm (Yedavalli et al., 2008). CID 44586781 was effective in suppressing HIV-1 replication in macrophages and T cells without imparting significant cytotoxicity in vivo. Notably, DDX3 was one of the first helicases subjected to the structurebased design of small molecular inhibitors using a molecular modeling approach (Maga et al., 2008). Using the crystal structure of DDX3 bound with AMP, a potential inhibitor of the enzyme's ATPase activity, designated RBD4 (Table 1), was identified by pharmacophoric modeling and subsequent molecular dockingbased virtual screening of compound libraries. In cell-based assays, the small molecule inhibitor was found to be effective in inhibiting HIV-1 replication, thereby strengthening the power of the docking approach. Optimized inhibitors that interfere with DDX3 RNA binding and helicase activity were also identified by precise homology modeling followed by high-throughput molecular docking (Radi et al., 2012). Although there are other cellular helicases which could serve as potential anti-HIV1 drug targets, only DDX3 has been successfully targeted by small molecule inhibitors so far. Therefore, structure-based design and virtual screening approaches targeting additional helicases 
involved in HIV-1 replication may aid in the development of more potent and effective inhibitors.

\section{NS3}

The Hepatitis C virus (HCV) NS3 helicase plays a key role in HCV replication and has been an attractive target for developing antiviral drugs (Frick, 2007). A potent inhibitor of NS3 helicase was successfully identified by exploiting its crystal structure using molecular docking-based virtual screening (Chen et al., 2009). The blue soluble HT dye that docked into the NS3 ATP binding site was found to inhibit its helicase activity as measured by a FRET-based assay (Chen et al., 2009). The co-crystal structure of the compound with NS3 was subjected to a small chemical fragment-based virtual screening search, leading to the discovery of a novel triphenylmethane derivative (Compound 12) that suppressed HCV replication in host cells (Chen et al., 2009). More recently, an in silico small molecule docking screen was used to identify an anti-helminthic drug (ivermectin) as a potent inhibitor of NS3 helicase activity; furthermore, ivermectin suppressed replication of common flaviviruses in cultured cells (Mastrangelo et al., 2012).

\section{DNA2}

The crystal structure of murine DNA2 bound to a short (15 nt) single-stranded DNA molecule revealed a unique mechanism of nucleolytic processing of DNA strand in which single-stranded DNA threaded through a central tunnel where it is bound by both the nuclease and helicase domains (Zhou et al., 2015). Although this murine DNA2 crystal structure has not yet been exploited for molecular docking of small molecules, an alternative virtual screening approach was used (Liu et al., 2016). The researchers employed the crystal structure of yeast Upf1RNA U15 complex and human Upf1-ADP complex (because they share high sequence identity with DNA2) to generate a stable homology model of human DNA2 bound to single-stranded DNA and then predicted potential druggable sites on the protein surface by docking a set of FDA-approved drug molecules. The most favorable docking pocket with the maximum score and DNA binding affinity was then subjected to HTS using a large NCI Developmental Therapeutics Program (DTP) library of small molecules. The approach led to the successful discovery of a lead compound (C5) that was predicted to bind to the DNA binding sites within the helicase domain.

The helicase domain-interacting molecule, designated C5 (Table 1), impaired nuclease, ATPase, and helicase activities of DNA2 (Liu et al., 2016). C5 inhibited proliferation of multiple cancer cell lines originating from breast, colon, prostate, or lung. Depletion of DNA2 in the breast cancer cell line MCF7 suppressed the anti-proliferative effect of $\mathrm{C} 5$, suggesting that DNA2 is the target of the compound in vivo. Consistent with this finding, embryonic stem cells from dna $2^{-/-}$mice were also resistant to C5. The authors did not assess if C5 caused sequestration of DNA2 on DNA, but this would be a worthwhile experiment to address the cytotoxicity of the DNA2-interacting compound.

In further cell-based assays, C5 was shown to inhibit singlestranded DNA annealing and HR, and this effect was likely due to a negative effect on DNA2-mediated end resection because RPA foci formation was reduced after CPT exposure in the C5treated cells (Liu et al., 2016). To assess the effect of C5 on DNA2's involvement in fork stabilization/restart, DNA fiber assays were performed with cells exposed to the replication inhibitor $\mathrm{HU}$ or low levels of CPT. The results from these assays indicated that C5 prevents normal restart of stalled replication forks. Furthermore, C5 prevented over-resection of stalled forks, suggesting the compound prevents DNA2's catalytic activities from processing stalled or regressed forks. Finally, it was shown that C5 sensitized cancer cells lines to various chemotherapeutic agents including a PARP inhibitor and CPT.

\section{NEW DNA HELICASE STRUCTURES PROVIDE FUTURE TARGETS FOR MOLECULAR DOCKING}

Recent discoveries of DNA helicase crystal structures have been informative from a mechanistic perspective and suggest that the development of specific helicase inhibitors using rational drug design approaches will accelerate in the future. Given the importance of RecQ helicases in genomic stability and their proposed differences and overlap in function, efforts to dock compounds on functionally distinct and less conserved domains of RecQ helicases is warranted and may provide useful tools to not only explore RecQ biological functions but also develop chemotherapy drugs against the helicase targets. In the following sections, we will discuss some key structural features of RecQ helicases and a Fe-S helicase (XPD) which may be exploited for drug development.

\section{RECQL1}

Like the other RecQ helicases, RECQL1 possesses two conserved RecA motor domains positioned such that the nucleotide binds within the cleft (Pike et al., 2009, 2015; Lucic et al., 2011) (Figure 3). High-resolution X-ray crystal structures of human RECQL1 bound to DNA and biochemical studies by the Gileadi and Vindigni labs revealed that the conserved $\mathrm{WH}$ domain (adjacent to the $\mathrm{Zn}^{2+}$ binding domain) bears a prominent $\beta$ hairpin structure with a tyrosine residue (Y564) at the tip which acts as a unique strand separating pin; this $\beta$-hairpin is also found in WRN and BLM helicases, but theirs are considerably shorter than the one located in RECQL1 (Pike et al., 2015). The intimate interaction of the RECQL1 strand-separating $\beta$-hairpin with the DNA branchpoint of the single-stranded DNA-double-stranded DNA junction suggests a relevant target for molecular docking by small molecules. Nonetheless, conservation of the $\beta$-hairpin among other DNA helicases may compromise its utility as a drug target due to specificity issues. However, RECQL1's strandseparating pin is buttressed by a protein dimer interface required for optimal duplex DNA unwinding; furthermore, RECQL1 also forms a tetramer that is implicated in $\mathrm{HJ}$ branch-migration. We conjecture that small molecules which dock at sites of critical contact points for oligomerization (e.g., dimer interface) might effectively modulate RECQL1's assembly state that would have dramatic consequences for biochemical and cellular function 


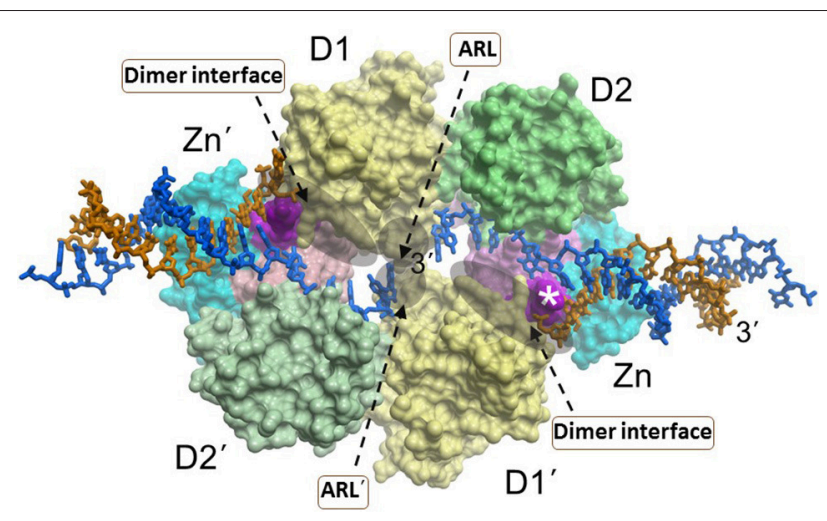

FIGURE 3 | A dimer of RECQL1 molecules, bound to two forked DNA molecules. The first RECQ1 monomer is marked with standard lettering, the second monomer is with primes. The two RecA binding domains (D1, D2) and $\mathrm{Zn}^{2+}$ binding domain $(\mathrm{Zn})$ are indicated. The white asterisk denotes the strand-separating beta hairpin. 3' end of DNA strand is indicated. The locations of the conserved aromatic-rich loops (ARL) implicated in the coupling of single-stranded DNA binding to ATP hydrolysis are indicated by the gray shadow. The region comprising the dimer interface between RECQL1 monomers is indicated by gray shadow. Docking small molecules at the ARL or dimer interface may provide a strategy to modulate RECQL1's catalytic function. Image was modified from one kindly provided by Dr. Opher Gileadi, University of Oxford.

(Figure 3). In addition, RECQL1 has a conserved aromaticrich loop (ARL) within the ATPase/helicase core domain that couples single-stranded DNA binding to ATP hydrolysis; the critical nature of RECQL1's ARL for its helicase activity, as revealed by site-directed mutagenesis studies (Banerjee et al., 2015), suggests another structural target to pharmacologically modulate its catalytic activity (Figure 3). However, the fact that all five human RecQ helicases possess the conserved ARL (Estep and Brosh, 2017) raises doubt if interaction specificity of a small molecule would be easily achievable unless mitigating factors are addressed with sophisticated molecular docking approaches (see below).

\section{BLM}

A recent BLM-DNA crystal structure solved by the Gileadi lab provided fresh insight to its DNA unwinding mechanism, suggesting a base-flipping action that is critical for duplex strand separation (Newman et al., 2015) (Figure 4). The mobility of the $\mathrm{WH}$ domain evident from the BLM structures suggests that a small molecule which docks in an allosteric site controlling the relative orientation may alter BLM's DNA unwinding mechanism. In addition, a new significance to the auxiliary HRDC domain found only in the human BLM and WRN helicases was ascribed. The HRDC domain was previously implicated in specialized DNA substrate recognition/binding and protein interaction for BLM and WRN [for review, see (Estep and Brosh, 2017)]. Moreover, the BLM HRDC domain is functionally important in double $\mathrm{HJ}$ dissolution, a reaction catalyzed by a BLM-topoisomerase complex that is believed to help suppress $\mathrm{SCE}$, a characteristic feature of Bloom's syndrome (Wu et al.,
2005). The new BLM structural data indicated a close residence of the HRDC domain to the nucleotide-binding pocket formed by the cleft between the two RecA domains (Newman et al., 2015), suggesting a structural arrangement that might be affected by HRDC-interacting small molecules (Figure 4). Compounds that interfere with the interaction of the HRDC with the RecA cleft would be predicted to disrupt the overall catalytic ATPase cycle of BLM, which in turn would affect its helicase activity. Thus, the BLM-DNA structure provides a framework for rational design of BLM-specific inhibitors that should deeply perturb its mechanism of action in cells.

\section{RECQL4}

Hereditary mutations in RECQL4 result in three genetically distinct diseases known as Rothmund-Thomson syndrome, Baller-Gerold syndrome, and RAPADILINO syndrome (Lu et al., 2017). While the RECQL4 gene product is a DNA helicase, the unwinding activity catalyzed by the purified recombinant RECQL4 helicase protein measured in vitro on conventional duplex DNA substrates is relatively weak (Macris et al., 2006; Xu and Liu, 2009). The limited unwinding activity of RECQL4 may be due to its strong annealing activity and its protein architecture as it lacks the classical $\mathrm{Zn}^{2+}$ binding domain and $\mathrm{WH}$ domain found in most other RecQ helicases (Figure 5). However, as revealed by structural and biochemical studies from the Kisker lab, the C-terminus of RECQL4 contains a unique $\mathrm{Zn}^{2+}$ binding domain (R4ZBD) and a region sharing homology to two winged helices that are distinct from the RQC WH in other RecQs (Kaiser et al., 2017). The unique identity of RECQL4's C-terminal region, which was found to be important for DNA unwinding (Kaiser et al., 2017), suggests a potential site for molecular docking of small molecules in the upper or lower half of the R4ZBD-WH to modulate its catalytic function (Figure 5).

\section{RECQL5}

The most recently solved structure of a human RecQ helicase was that of RECQL5 (Newman et al., 2017) (Figure 6). This work from the Gileadi lab showed that RECQL5 binds $\mathrm{Zn}^{2+}$ via the conserved domain found in RECQL1, WRN, and BLM and that RECQL5 possesses a unique adjacent $\alpha$-helix with positively charged residues on its surface not found in the other human RecQ helicases. The unique RECQL5 $\alpha$-helix is proposed to operate as a wedge analogous to the $\beta$-hairpin in RECQL1, WRN, and BLM, suggesting a potential RECQL5-specific domain to target with small molecules (Figure 6). From a molecular docking perspective, it is quite interesting that RECQL5 was demonstrated to exist in two distinct conformations (open and closed) that are regulated by nucleotide binding. Further studies may identify interfacial small molecule inhibitors that bind within the inter-domain cleft and lock it into the open or closed conformation (Figure 6). Screening for small molecules that affect the nucleotide-induced conformational switch of RECQL5 may be informative for further understanding mechanism. The aforementioned $\alpha$-helix was implicated in DNA binding and sitespecific mutagenesis revealed that it plays an important role in helicase activity. RECQL5-interacting compounds that affect the conformational freedom of the $\alpha$-helix and other key structural 

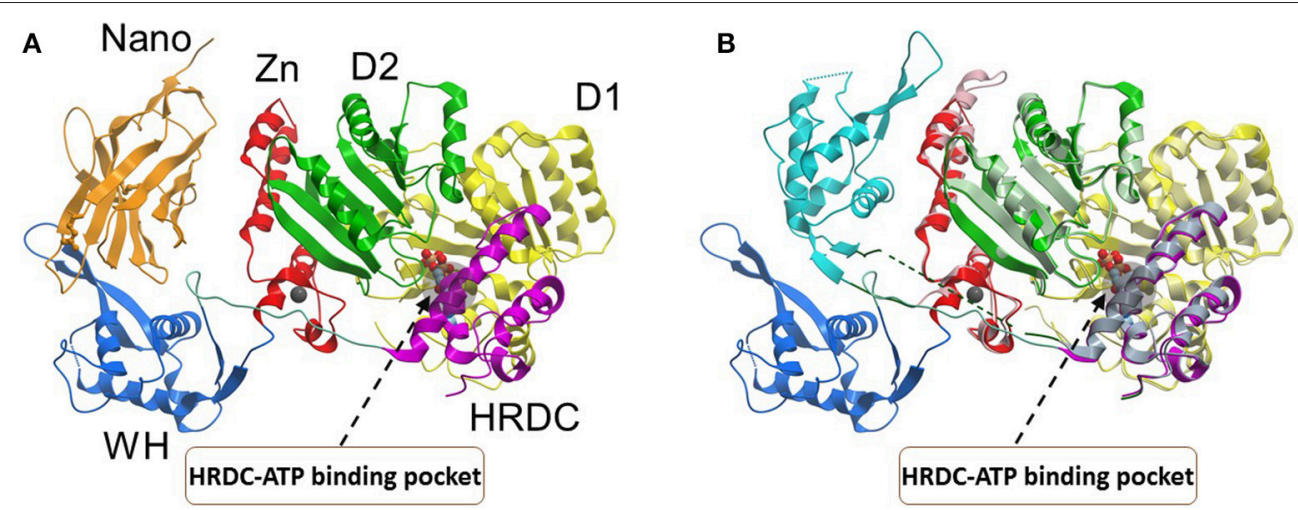

FIGURE 4 | Conformations of the BLM helicase core and winged helix domains. (A) A co-crystal structure with a nanobody (orange), which shifts the WH domain out of its helicase-active position (PDB:4CDG). (B) A superposition of the nanobody-bound conformation (WH domain in blue, nanobody omitted), with a DNA-bound conformation (WH domain in cyan, DNA omitted; PDB:4CGZ). The RecA domains (D1, D2) and the conserved HRDC are indicated with a region of the HRDC residing close to the ATP binding pocket shown by gray shadow. Small molecules which bind to the HRDC may modulate ATP binding and/or hydrolysis by BLM. Image was modified from one kindly provided by Dr. Opher Gileadi, University of Oxford.

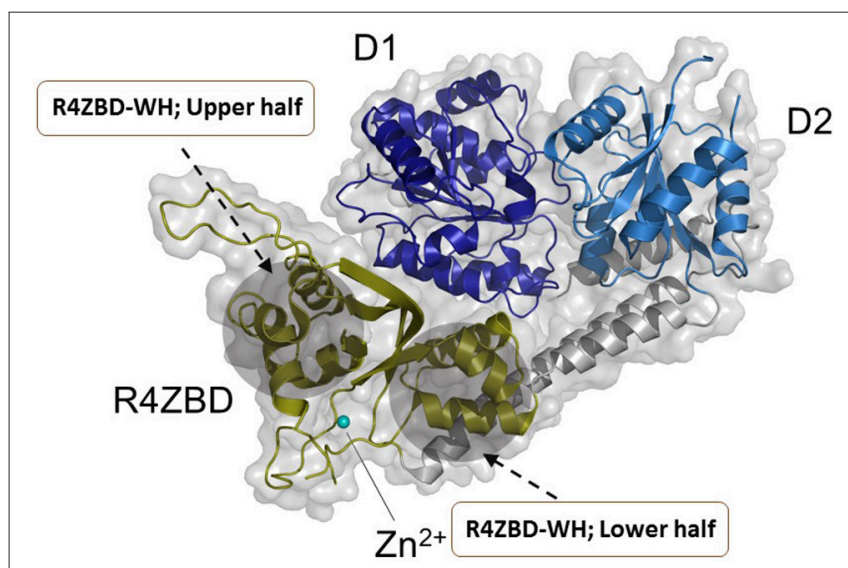

FIGURE 5 | Structure of human RECQL4 (residues 449-1111). The ATPase domain, comprising HD1 and HD2, are shown in dark blue and light blue, respectively. RECQL4 features a structurally unique domain, termed RECQL4-Zn²+-binding domain (R4ZBD), shown in olive. The R4ZBD coordinates a $\mathrm{Zn}^{2+}$-ion (cyan sphere). The gray shadow regions represent the upper and lower halves of the R4ZBD that may be suitable for molecular docking of small molecules to the unique $\mathrm{Zn}^{2+}$ binding domain of RECQL4. RECQL4 harbors the SId2-homology domain at its N-terminus (not shown). Image was modified from one kindly provided by Drs. Sebastian Kaiser and Caroline Kisker, University of Wuerzburg.

elements would be anticipated to impact its catalytic functions and potentially modulate its functions in cells.

\section{XPD}

Eukaryotic XPD is a component of the general transcription factor (TF)IIH complex that is implicated in both cellular transcription and nucleotide excision repair (NER) of damaged DNA (Kraemer et al., 2007). Mutations in the XPD gene are linked to genetic diseases characterized by premature aging and/or cancer predisposition including Trichothiodystrophy (TTD), Xeroderma pigmentosum, and Cockayne's syndrome
(Lehmann, 2001). High-resolution crystal structures of XPD helicase and the data obtained from the associated biochemical and mutational studies over the past decade provided significant mechanistic insights about the function of this important class of Fe-S helicases (Liu et al., 2008; Wolski et al., 2008; Kuper et al., 2012; Abdulrahman et al., 2013). Crystal structures of archaeal homologs of XPD revealed that in addition to two canonical RecA motor domains, the structure contains a $\mathrm{Fe}-\mathrm{S}$ cluster and Arch domain. In its proper conformational state, the XPD Fe-S cluster remains tightly connected to the ATP binding/hydrolysis domain. It has been proposed that the wedgelike structure formed by the Fe-S and Arch domains facilitates unwinding of duplex DNA during ATP-driven translocation of the enzyme. Furthermore, in addition to its essential role in helicase activity, mutational studies confirmed that Fe-S cluster is structurally important to maintain proper folding and stability of the XPD protein (Rudolf et al., 2006; Fan et al., 2008; Pugh et al., 2008).

More functional insights came from the crystal structure of XPD from Thermoplasma acidophilium (taXPD) in complex with DNA solved by the Kisker lab (Kuper et al., 2012) (Figure 7). The taXPD-DNA complex structure, combined with biochemical and mutational analyses from their lab (Kuper et al., 2012) and Spies' (Pugh et al., 2012), has begun to elucidate the underlying mechanism for XPD's DNA translocation polarity, thereby providing insight into the role of the helicase during NER. Apart from the Fe-S cluster domain, the Arch domain of XPD also has been shown to be critical for its DNA binding and strand separating activities. Introduction of a TTD-linked point mutation (XPD-C259Y) or deletion of the entire Arch domain (XPD- $\triangle \mathrm{ARCH}$ ) was found to impair DNA binding and helicase activity of XPD (Abdulrahman et al., 2013). Collectively, these studies suggest that Fe-S cluster and Arch domain play key roles in the unwinding mechanism and governing XPD functions during DNA damage repair. Targeting the Arch and Fe-S domains of XPD with small molecules may be valuable (Figure 7); 


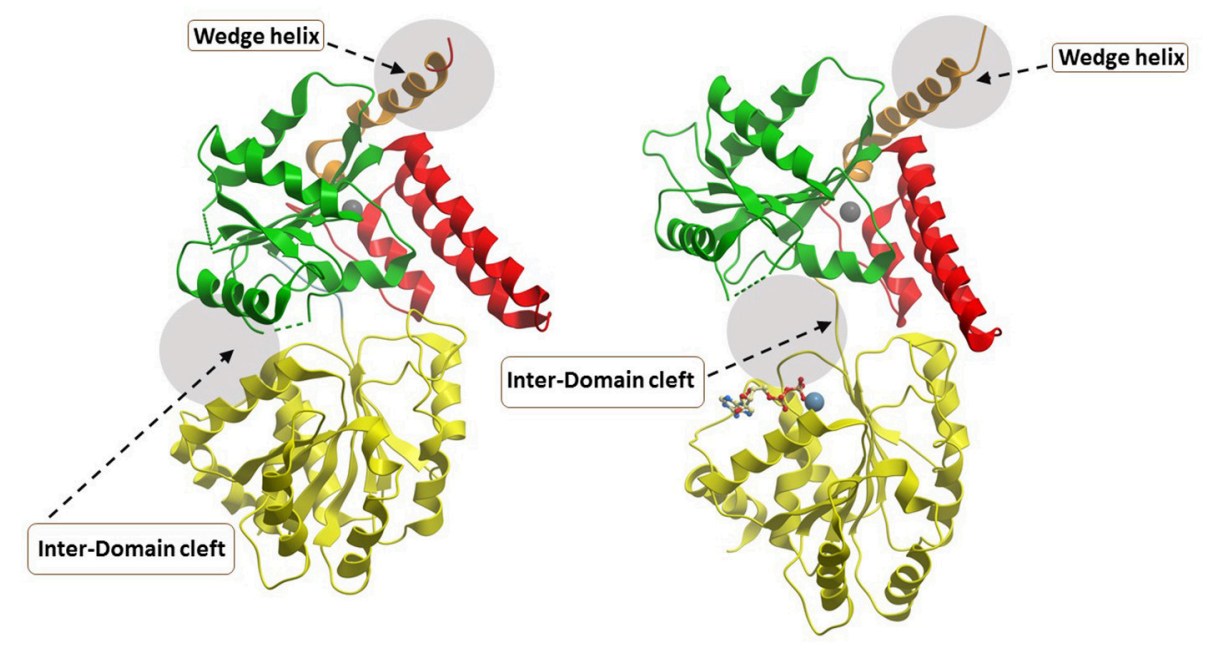

FIGURE 6 | Closed and open conformations of RECQL5 helicase domain. (A) Closed state, no nucleotide (PDB ID: 5LB8); (B) Open state, with Mg2+-ADP (PDB: 5LB3). Note the absence of the WH and HRDC domains in the C-terminal region of the protein which can be found in WRN, BLM, and RECQL1. The unique "wedge" $\alpha$-helix of RECQL5 critical for helicase activity is indicated and may be targeted by small molecules to modulate ATP-dependent strand separation. Also indicated by gray zone is the inter-domain cleft which widens significantly between the open and closed conformations. The inter-domain cleft may be a useful site for molecular docking of small molecule interfacial compounds that perturb open-closed conformational switches of RECQL5. Image was modified from one kindly provided by Dr. Opher Gileadi, University of Oxford.

however, these domains are conserved in other Fe-S helicases (FANCJ, RTEL1, DDX11), raising doubt if they would be specific. Nonetheless, given that DNA damaging chemotherapy drugs often introduce bulky lesions recognized by NER, it is reasonable to postulate that small molecule targeted inhibition of DNA unwinding by the XPD helicase (thought to be an early sensor or verifier of the DNA damage) would be useful to sensitize tumors to certain compounds used in anti-cancer treatments.

Further research in this area to solve structures of XPD with key DNA structural intermediates (e.g., DNA bubbles with sitespecific damage), as well as structures of other Fe-S helicases, is likely to advance efforts in molecular docking and HTS for small molecules that modulate their functions. With the discovery of specific and potent inhibitors of Fe-S helicases, the co-crystal structures of helicase-DNA-small molecule complexes may elucidate key helicase interactions in DNA metabolic pathways. Molecular docking-based virtual screening should also be considered as a highly effective complementary approach toward the discovery of small molecule inhibitors of diseaselinked DNA repair helicases.

\section{CHALLENGES TO DEVELOPMENT AND APPLICATION OF HELICASE INHIBITORS}

Although small molecule inhibitors of a few viral helicases showed preclinical and clinical success [e.g., the herpes simplex virus helicase-primase inhibitor Amenamevir (ASP2151) (Chono et al., 2010) and other drug candidates (Kleymann et al., 2002)], development of highly specific and pharmacologically effective helicase inhibitors is still challenging. In general, high throughput

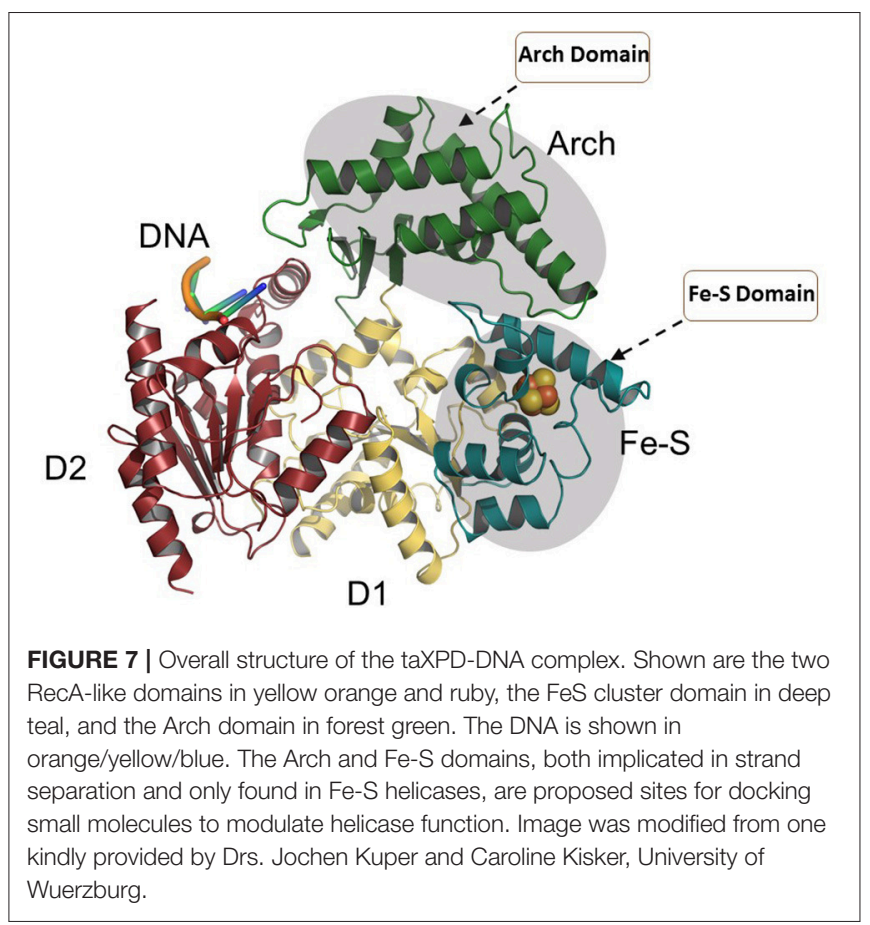

biochemical screening assays return very few hits and most often they fail to deliver expected biological effects in subsequent cellbased assays or display poor pharmacological outcomes [e.g., HPV helicase inhibitor (Faucher et al., 2004)]. As is the case for a significant number of small molecule inhibitors, bioavailability is likely to be one of the major obstacles to developing clinically effective DNA repair helicase inhibitors and target validation is 


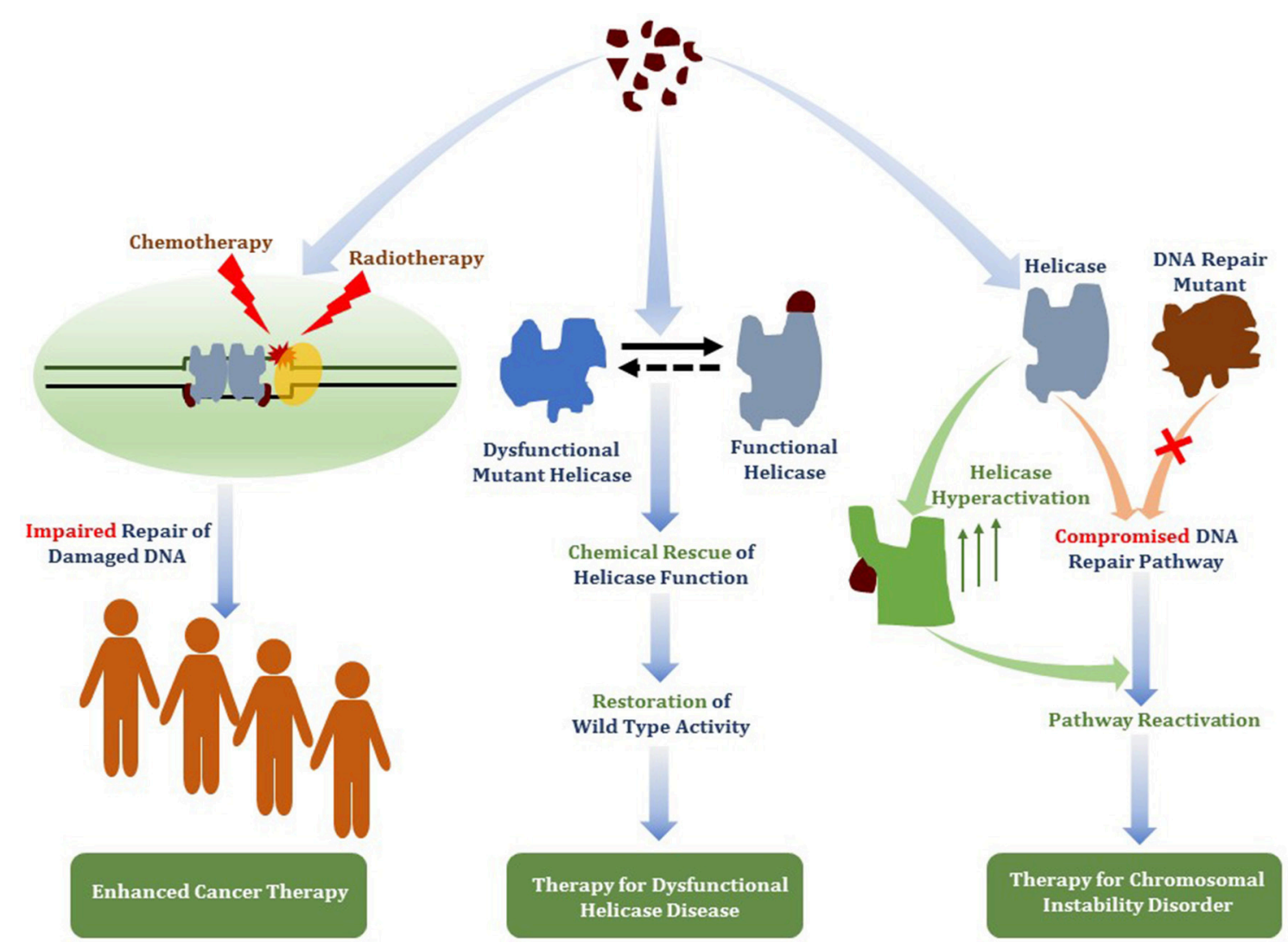

FIGURE 8 | Therapeutic avenues for helicase-interacting small molecules. Compounds that bind DNA helicase proteins may be useful for (i) targeted helicase inhibition to deter DNA damage response pathways to enhance chemotherapy drug or radiation strategies (ii) chemical rescue of catalytically defective and/or misfolded helicase proteins due to missense mutations linked to hereditary chromosomal instability disorders, or (iii) functional activation of a wild-type helicase that has an overlapping role with a DNA repair pathway that is defective due to a genetic inactivating mutation linked to a DNA repair disorder.

required (Bunnage et al., 2013). Therefore, cellular bioavailability parameters including inhibitor aqueous solubility, nonspecific binding to the cell membrane and extracellular matrix, cellular uptake, intracellular metabolic stability, and biotransformation to an inactive secondary metabolite should be taken into consideration for the successful therapeutic exploitation of a newly identified promising drug (Frye, 2010; Workman and Collins, 2010), such as a lead molecule against a helicase protein. For example, although BLM inhibitors ML216 and its analog 33 were found to exhibit good general pharmacokinetic properties including $\operatorname{cog} \mathrm{P}$ (computational method for measurement of drug hydrophilicity and lipophilicity properties), microsomal stability, and plasma stability, both compounds displayed low solubility and permeability suggesting that further optimization of the lead compounds is required (Rosenthal et al., 2013). In this scenario, sensitive assays such as a HPLC-MS based method to determine bioavailability of a given compound inside cells may prove fruitful to assess cellular uptake of helicase inhibitors (Teuscher et al., 2017).

Given the precise functions of helicases in nucleic acid metabolism, another consideration is if the helicase-interacting compound reaches its desired subcellular localization (i.e., nucleus, cytosol, mitochondrion) to bind its desired target helicase inside the respective cellular compartment. In order to get the most desirable therapeutic effect and to minimize the negative side effects, it is also very important to ensure that the helicase inhibitors are delivered specifically to their sites of action within the cells. Nuclear targetted delivery of these small molecule inhibitors might be achieved using nanoparticles coated with nuclear localization signal (NLS) (Kang et al., 2010a). Similarly, clinically approved nanoparticles should be considered to deliver the helicase inhibitors selectively to the target tumor sites at sufficient concentration to attain therapeutic efficacy.

One of the prime challenges associated with the development of helicase inhibitors is their relative potency and specificity. The inhibitors should be potent enough to exert their biological effects at minimal concentration. For example, Mcm2-7 helicase inhibitor ciprofloxacin (Simon et al., 2013) and BLM helicase inhibitor ML216 (Nguyen et al., 2013) were found to exhibit their bioactivity at relatively high concentrations $(160-350 \mu \mathrm{M}$ and $50 \mu \mathrm{M}$, respectively; see Table 1 ). Therefore, structural optimization of these hit compounds is warranted to obtain more potent leads. Moreover, the effect of ML216 is not entirely specific to BLM helicase because the compound also inhibits DNA 
unwinding by the sequence-related WRN helicase with a similar potency (Nguyen et al., 2013). Hence, promising hits identified for a target helicase from the initial screening should be further assessed for their potency as a function of drug concentration as well as for their specificity by determining their effects on other helicases. Substantial counterscreening of the newly identified potential helicase inhibitors is required before they can be reliably pursued in preclinical models.

A number of drug resistance mechanism are known to operate which contribute to tumor resistance (Holohan et al., 2013). These include: (i) drug efflux or alteration by activation or inactivation; (ii) alterations in the drug target by mutation or change in gene expression; (iii) repair of chemotherapy- or radiation-induced DNA damage; (iv) up-regulation or activation of compensatory signaling pathways; (v) cell death evasion (e.g., attenuated apoptosis). From the perspective of DNA helicase inhibitors, one of the basic principles is to compromise a helicase-dependent pathway of repair [(iii) above] to confer synthetic lethality; however, it is possible that another repair pathway or signaling pathway is elicited that compromises the efficacy of the helicase inhibitor. Certainly, it is plausible that even the functional redundancy between members of the RecQ or Fe-S helicases, for example, may contribute to helicase inhibitor resistance by a compensatory overlapping pathway. Other avenues of drug resistance, such as those mentioned above, may allow resistance to the anti-cancer effects of a helicasedirected drug inhibitor; however, little is known in this area because it is such a new field. The fact that tumors are often heterogeneous may allow cancer cell subpopulations survive under pressure from a cancer drug (Zahreddine and Borden, 2013), including one against a specific DNA helicase. These topics all deserve prioritized attention.

In terms of molecular docking approaches for the discovery of compounds that inhibit helicases and other DNA repair proteins, a significant challenge is the protein flexibility of the target in which intrinsic conformational states may compromise a good fit for the docking ligand (Tripathi and Bankaitis, 2017). Other mitigating factors including protein pocket architecture to accommodate the three-demonsional geometry of the ligand, ligand access (surface or protein interior), the role of structured water molecules in the ligandtarget interaction, protonation, and ionization states of the protein: ligand system, and entropy considerations. Advances in artificial intelligence and machine learning algorithms provide new and innovative direction for structure-based drug design. These efforts, combined with high-resolution structures of helicase proteins, provide excitement for anticipated progress.

\section{SUMMARY AND FUTURE DIRECTIONS}

DNA helicases are often recruited to sites of DNA damage or stalled replication forks. The very nature of their catalytic function to separate complementary DNA strands is imperative to a wide variety of DNA transactions that play instrumental roles in cellular DNA replication, recombination, repair, and transcription. Therefore, chemical modulation of the molecular functions of DNA helicases provides an approach to alter not only the efficiency or fidelity of transactions in nucleic acid metabolism but also affect cellular homeostasis, including the division rate of cancerous cells. As detailed in this review, DNA helicases join a larger class of DNA metabolic proteins that are considered as potential targets to augment radiation and chemotherapy strategies to combat cancer. Small moleculeinduced trapping of DNA helicases may represent a generalized mechanism exemplified by certain topoisomerase and PARP inhibitors that exert poisonous consequences, especially in rapidly dividing cancer cells. An area that remains underexplored is the synergism between compounds that modulate different DNA proteins. This is particularly interesting from the perspective of DNA helicase inhibitors that might be combined with compounds that deter the functions of other DNA repair enzymes to enact targeted anti-proliferative and lethal effects in various cancer types (Figure 8). Just as chemical and genetic synthetic lethality has become more widely appreciated and better understood, we expect that pharmacological modulation of helicase function will move to the forefront as molecular motor DNA unwinding enzymes play such pivotal roles in nucleic acid metabolism and cross-talk with many cellular pathways. With the increased knowledge of structure-activity relationships from the solution of helicase structures, we anticipate that molecular modeling will provide a more readily accessible and informed pathway for the discovery of novel helicaseinteracting compounds. An important challenge in the field will be the utilization of helicase-modulating drugs in preclinical models that will accelerate their implementation in therapeutic approaches.

A unique aspect of this review is to detail potential strategies to target helicase with small molecules using a structure-design molecular docking approach (Figure 2). We believe that virtual screening of small molecule libraries to identify compounds predicted to modulate helicase function will become main-streamed as more helicase structures (and their conformational states induced by ligand binding) become solved and computational strategies advance. An illustrative example of this approach was recently provided by the Berger lab. Lawson et al. observed that the structural interactions of nucleic acid or the antibiotic bicyclomycin with the same binding site in the hexameric RNA translocase/helicase Rho are distinguished from each other by the closed-ring (translocase competent) vs. open-ring (RNA binding defective) conformations of Rho, respectively (Lawson et al., 2016). This work is significant because it showed that nucleic acid substrate loading by a helicase could be modulated by a small molecule via a conformational switch in the enzyme that altered its ring-closure dynamics. Furthermore, the Rho-bicyclomycin study leads to the anticipation that other helicase-interacting small molecules may be identified virtually from compound libraries using a molecular docking approach that would be highly selective and mechanistically driven.

Although molecular compound inhibitors of DNA helicases or more generally DNA repair enzymes are increasingly discussed, conversations and research directions could also be directed toward small molecule chemical rescue of 
catalytically dysfunctional or misfolded helicase proteins as well as activation or up-regulation of wild-type helicase-catalyzed strand separation (Figure 8). Given the number of helicase missense mutations linked to hereditary disorders or associated with cancer (Suhasini and Brosh, 2013), and following the lead of other clinically relevant targets [e.g., chemical rescue of p53 missense mutations (Bullock and Fersht, 2001)], the prospect of finding treatments or cures for certain chromosomal instability disorders arising from catalytic deficiencies in helicase proteins is plausible. In another realm, helicase activation may have therapeutic value. For example, the possible functional overlap of DNA helicases [e.g., RecQ family members (Brosh, 2013; Croteau et al., 2014)] suggests that increased activity of one helicase may help to overcome the deficiency of another helicase or DNA repair protein (Figure 8). With molecular docking

\section{REFERENCES}

Abdulrahman, W., Iltis, I., Radu, L., Braun, C., Maglott-Roth, A., Giraudon, C., et al. (2013). ARCH domain of XPD, an anchoring platform for CAK that conditions TFIIH DNA repair and transcription activities. Proc. Natl. Acad. Sci. U.S.A. 110, E633-E642. doi: 10.1073/pnas.1213981110

Abid Ali, F., and Costa, A. (2016). The MCM helicase motor of the eukaryotic replisome. J. Mol. Biol. 428(9 Pt B), 1822-1832. doi: 10.1016/j.jmb.2016.01.024

Aggarwal, M., Banerjee, T., Sommers, J. A., and Brosh, R. M. Jr. (2013a). Targeting an Achilles' heel of cancer with a WRN helicase inhibitor. Cell Cycle 12, 3329-3335. doi: 10.4161/cc.26320

Aggarwal, M., Banerjee, T., Sommers, J. A., Iannascoli, C., Pichierri, P., Shoemaker, R. H., et al. (2013b). Werner syndrome helicase has a critical role in DNA damage responses in the absence of a functional fanconi anemia pathway. Cancer Res. 73, 5497-5507. doi: 10.1158/0008-5472.can-12-2975

Aggarwal, M., Sommers, J. A., Shoemaker, R. H., and Brosh, R. M. Jr. (2011). Inhibition of helicase activity by a small molecule impairs Werner syndrome helicase (WRN) function in the cellular response to DNA damage or replication stress. Proc. Natl. Acad. Sci. U.S.A. 108, 1525-1530. doi: $10.1073 /$ pnas. 1006423108

Aparicio, T., Guillou, E., Coloma, J., Montoya, G., and Mendez, J. (2009). The human GINS complex associates with Cdc45 and MCM and is essential for DNA replication. Nucleic Acids Res. 37, 2087-2095. doi: 10.1093/nar/gkp065

Awate, S., and Brosh, R. M. Jr. (2017). Interactive Roles of DNA Helicases and Translocases with the Single-Stranded DNA Binding Protein RPA in Nucleic Acid Metabolism. Int. J. Mol. Sci. 18:E1233. doi: 10.3390/ijms18061233

Banerjee, T., Aggarwal, M., Sommers, J. A., and Brosh, R. M. Jr. (2016). Biochemical and cell biological assays to identify and characterize DNA helicase inhibitors. Methods 108, 130-141. doi: 10.1016/j.ymeth.2016.04.007

Banerjee, T., Sommers, J. A., Huang, J., Seidman, M. M., and Brosh, R. M. Jr. (2015). Catalytic strand separation by RECQ1 is required for RPA-mediated response to replication stress. Curr. Biol. 25, 2830-2838. doi: $10.1016 /$ j.cub.2015.09.026

Belon, C. A., and Frick, D. N. (2009). Helicase inhibitors as specifically targeted antiviral therapy for hepatitis C. Future Virol. 4, 277-293. doi: 10.2217/fvl.09.7

Brosh, R. M., Jr. (2013). DNA helicases involved in DNA repair and their roles in cancer. Nat. Rev. Cancer 13, 542-558. doi: 10.1038/nrc3560

Bryant, H. E., Schultz, N., Thomas, H. D., Parker, K. M., Flower, D., Lopez, E., et al. (2005). Specific killing of BRCA2-deficient tumours with inhibitors of poly(ADP-ribose) polymerase. Nature 434, 913-917. doi: 10.1038/nature03443

Budd, M. E., Choe, W. C., and Campbell, J. L. (1995). DNA2 encodes a DNA helicase essential for replication of eukaryotic chromosomes. J. Biol. Chem. 270, 26766-26769.

Budd, M. E., Choe, W., and Campbell, J. L. (2000). The nuclease activity of the yeast DNA2 protein, which is related to the RecB-like nucleases, is essential in vivo. J. Biol. Chem. 275, 16518-16529. doi: 10.1074/jbc.M909511199 and high-throughput screens becoming more commonplace, the hypothesis that up-regulation of catalytic function by a helicase or DNA repair enzyme can rescue a helicase-deficient disease state may become testable in cell-based and pre-clinical models.

\section{AUTHOR CONTRIBUTIONS}

$\mathrm{AD}$ and $\mathrm{RB}$ both contributed to writing original component and editing of the manuscript.

\section{ACKNOWLEDGMENTS}

This work was supported by the Intramural Research Program of the National Institutes of Health, National Institute on Aging.

Budd, M. E., Reis, C. C., Smith, S., Myung, K., and Campbell, J. L. (2006). Evidence suggesting that Pif1 helicase functions in DNA replication with the Dna2 helicase/nuclease and DNA polymerase delta. Mol. Cell. Biol. 26, 2490-2500. doi: $10.1128 / \mathrm{mcb} \cdot 26.7 .2490-2500.2006$

Bullock, A. N., and Fersht, A. R. (2001). Rescuing the function of mutant p53. Nat. Rev. Cancer 1, 68-76. doi: 10.1038/35094077

Bunnage, M. E., Chekler, E. L., and Jones, L. H. (2013). Target validation using chemical probes. Nat. Chem. Biol. 9, 195-199. doi: 10.1038/nchembio.1197

Chang, S., Multani, A. S., Cabrera, N. G., Naylor, M. L., Laud, P., Lombard, D., et al. (2004). Essential role of limiting telomeres in the pathogenesis of Werner syndrome. Nat. Genet. 36, 877-882. doi: 10.1038/ng1389

Chen, C. S., Chiou, C. T., Chen, G. S., Chen, S. C., Hu, C. Y., Chi, W. K., et al. (2009). Structure-based discovery of triphenylmethane derivatives as inhibitors of hepatitis C virus helicase. J. Med. Chem. 52, 2716-2723. doi: $10.1021 / \mathrm{jm} 8011905$

Chong, J. P., Hayashi, M. K., Simon, M. N., Xu, R. M., and Stillman, B. (2000). A double-hexamer archaeal minichromosome maintenance protein is an ATP-dependent DNA helicase. Proc. Natl. Acad. Sci. U.S.A. 97, 1530-1535. doi: 10.1073/pnas.030539597

Chono, K., Katsumata, K., Kontani, T., Kobayashi, M., Sudo, K., Yokota, T., et al. (2010). ASP2151, a novel helicase-primase inhibitor, possesses antiviral activity against varicella-zoster virus and herpes simplex virus types 1 and 2. J. Antimicrob. Chemother. 65, 1733-1741. doi: 10.1093/jac/dkq198

Croteau, D. L., Popuri, V., Opresko, P. L., and Bohr, V. A. (2014). Human RecQ helicases in DNA repair, recombination, and replication. Annu. Rev. Biochem. 83, 519-552. doi: 10.1146/annurev-biochem-060713-035428

Crouch, J. D., and Brosh, R. M. Jr. (2017). Mechanistic and biological considerations of oxidatively damaged DNA for helicase-dependent pathways of nucleic acid metabolism. Free Radic. Biol. Med. 107, 245-257. doi: $10.1016 /$ j.freeradbiomed.2016.11.022

Cseh, A. M., Fabian, Z., Sumegi, B., and Scorrano, L. (2017). Poly(adenosine diphosphate-ribose) polymerase as therapeutic target: lessons learned from its inhibitors. Oncotarget 8, 50221-50239. doi: 10.18632/oncotarget.16859

de Renty, C., and Ellis, N. A. (2017). Bloom's syndrome: Why not premature aging?: a comparison of the BLM and WRN helicases. Ageing Res. Rev. 33, 36-51. doi: 10.1016/j.arr.2016.05.010

Du, X., Shen, J., Kugan, N., Furth, E. E., Lombard, D. B., Cheung, C., et al. (2004). Telomere shortening exposes functions for the mouse Werner and Bloom syndrome genes. Mol. Cell. Biol. 24, 8437-8446. doi: $10.1128 / \mathrm{mcb} .24 .19 .8437-8446.2004$

Estep, K. N., and Brosh, R. M. Jr. (2017). RecQ and Fe-S helicases have unique roles in DNA metabolism dictated by their unwinding directionality, substrate specificity, and protein interactions. Biochem. Soc. Trans. 46, 77-95. doi: 10.1042/bst20170044

Fan, L., Fuss, J. O., Cheng, Q. J., Arvai, A. S., Hammel, M., Roberts, V. A., et al. (2008). XPD helicase structures and activities: insights into the 
cancer and aging phenotypes from XPD mutations. Cell 133, 789-800. doi: 10.1016/j.cell.2008.04.030

Farmer, H., McCabe, N., Lord, C. J., Tutt, A. N., Johnson, D. A., Richardson, T. B., et al. (2005). Targeting the DNA repair defect in BRCA mutant cells as a therapeutic strategy. Nature 434, 917-921. doi: 10.1038/nature03445

Faucher, A. M., White, P. W., Brochu, C., Grand-Maitre, C., Rancourt, J., and Fazal, G. (2004). Discovery of small-molecule inhibitors of the ATPase activity of human papillomavirus E1 helicase. J. Med. Chem. 47, 18-21. doi: $10.1021 / \mathrm{jm} 034206 \mathrm{x}$

Fernandez-Millan, P., Lazaro, M., Cansiz-Arda, S., Gerhold, J. M., Rajala, N., Schmitz, C. A., et al. (2015). The hexameric structure of the human mitochondrial replicative helicase Twinkle. Nucleic Acids Res. 43, 4284-4295. doi: 10.1093/nar/gkv189

Frick, D. N. (2007). The hepatitis C virus NS3 protein: a model RNA helicase and potential drug target. Curr. Issues Mol. Biol. 9, 1-20.

Frye, S. V. (2010). The art of the chemical probe. Nat. Chem. Biol. 6, 159-161. doi: 10.1038/nchembio.296

Geronimo, C. L., and Zakian, V. A. (2016). Getting it done at the ends: Pifl family DNA helicases and telomeres. DNA Repair 44, 151-158. doi: 10.1016/j.dnarep.2016.05.021

Hengel, S. R., Malacaria, E., Folly da Silva Constantino, L., Bain, F. E., Diaz, A., Koch, B. G., et al. (2016). Small-molecule inhibitors identify the RAD52-ssDNA interaction as critical for recovery from replication stress and for survival of BRCA2 deficient cells. Elife 5:e14740. doi: 10.7554/eLife.14740

Hengel, S. R., Spies, M. A., and Spies, M. (2017). Small-Molecule Inhibitors Targeting DNA Repair and DNA repair deficiency in research and cancer therapy. Cell Chem. Biol. 24, 1101-1119. doi: 10.1016/j.chembiol.2017.08.027

Himaki, T., Masui, Y., Chono, K., Daikoku, T., Takemoto, M., Haixia, B., et al. (2012). Efficacy of ASP2151, a helicase-primase inhibitor, against thymidine kinase-deficient herpes simplex virus type 2 infection in vitro and in vivo. Antiviral Res. 93, 301-304. doi: 10.1016/j.antiviral.2011.11.015

Holohan, C., Van Schaeybroeck, S., Longley, D. B., and Johnston, P. G. (2013). Cancer drug resistance: an evolving paradigm. Nat. Rev. Cancer 13, 714-726. doi: $10.1038 /$ nrc3599

Huang, F., and Mazin, A. V. (2014). Targeting the homologous recombination pathway by small molecule modulators. Bioorg. Med. Chem. Lett. 24, 3006-3013. doi: 10.1016/j.bmcl.2014.04.088

Ishimi, Y., Sugiyama, T., Nakaya, R., Kanamori, M., Kohno, T., Enomoto, T., et al. (2009). Effect of heliquinomycin on the activity of human minichromosome maintenance 4/6/7 helicase. FEBS J. 276, 3382-3391. doi: $10.1111 / j .1742-4658.2009 .07064 . x$

Jia, P. P., Junaid, M., Ma, Y. B., Ahmad, F., Jia, Y. F., Li, W. G., et al. (2017). Role of human DNA2 (hDNA2) as a potential target for cancer and other diseases: a systematic review. DNA Repair 59, 9-19. doi: 10.1016/j.dnarep.2017.09.001

Kaiser, S., Sauer, F., and Kisker, C. (2017). The structural and functional characterization of human RecQ4 reveals insights into its helicase mechanism. Nat. Commun. 8:15907. doi: 10.1038/ncomms15907

Kang, B., Mackey, M. A., and El-Sayed, M. A. (2010a). Nuclear targeting of gold nanoparticles in cancer cells induces DNA damage, causing cytokinesis arrest and apoptosis. J. Am. Chem. Soc. 132, 1517-1519. doi: 10.1021/ja9102698

Kang, Y. H., Lee, C. H., and Seo, Y. S. (2010b). Dna2 on the road to Okazaki fragment processing and genome stability in eukaryotes. Crit. Rev. Biochem. Mol. Biol. 45, 71-96. doi: 10.3109/10409230903578593

Katsumata, K., Weinberg, A., Chono, K., Takakura, S., Kontani, T., and Suzuki, H. (2012). Susceptibility of herpes simplex virus isolated from genital herpes lesions to ASP2151, a novel helicase-primase inhibitor. Antimicrob. Agents Chemother. 56, 3587-3591. doi: 10.1128/aac.00133-12

Kleymann, G., Fischer, R., Betz, U. A., Hendrix, M., Bender, W., Schneider, U., et al. (2002). New helicase-primase inhibitors as drug candidates for the treatment of herpes simplex disease. Nat. Med. 8, 392-398. doi: 10.1038/nm0402-392

Korhonen, J. A., Gaspari, M., and Falkenberg, M. (2003). TWINKLE Has $5^{\prime}$ $>3^{\prime}$ DNA helicase activity and is specifically stimulated by mitochondrial single-stranded DNA-binding protein. J. Biol. Chem. 278, 48627-48632. doi: 10.1074/jbc.M306981200

Kraemer, K. H., Patronas, N. J., Schiffmann, R., Brooks, B. P., Tamura, D., and DiGiovanna, J. J. (2007). Xeroderma pigmentosum, trichothiodystrophy and Cockayne syndrome: a complex genotype-phenotype relationship. Neuroscience 145, 1388-1396. doi: 10.1016/j.neuroscience.2006.12.020
Kroemer, R. T. (2007). Structure-based drug design: docking and scoring. Curr. Protein Pept. Sci. 8, 312-328. doi: 10.2174/138920307781369382

Kumar, S., Peng, X., Daley, J., Yang, L., Shen, J., Nguyen, N., et al. (2017). Inhibition of DNA2 nuclease as a therapeutic strategy targeting replication stress in cancer cells. Oncogenesis 6:e319. doi: 10.1038/oncsis.2017.15

Kuper, J., Wolski, S. C., Michels, G., and Kisker, C. (2012). Functional and structural studies of the nucleotide excision repair helicase XPD suggest a polarity for DNA translocation. EMBO J. 31, 494-502. doi: 10.1038/emboj.2011.374

Kwong, A. D., Rao, B. G., and Jeang, K. T. (2005). Viral and cellular RNA helicases as antiviral targets. Nat. Rev. Drug Discov. 4, 845-853. doi: 10.1038/nrd1853

Lahaye, A., Stahl, H., Thines-Sempoux, D., and Foury, F. (1991). PIF1: a DNA helicase in yeast mitochondria. EMBO J. 10, 997-1007.

Laud, P. R., Multani, A. S., Bailey, S. M., Wu, L., Ma, J., Kingsley, C., et al. (2005). Elevated telomere-telomere recombination in WRN-deficient, telomere dysfunctional cells promotes escape from senescence and engagement of the ALT pathway. Genes Dev. 19, 2560-2570. doi: 10.1101/gad.1321305

Lawson, M. R., Dyer, K., and Berger, J. M. (2016). Ligand-induced and smallmolecule control of substrate loading in a hexameric helicase. Proc. Natl. Acad. Sci. U.S.A. 113, 13714-13719. doi: 10.1073/pnas.1616749113

Lehmann, A. R. (2001). The xeroderma pigmentosum group D (XPD) gene: one gene, two functions, three diseases. Genes Dev. 15, 15-23.

Liu, H., Rudolf, J., Johnson, K. A., McMahon, S. A., Oke, M., Carter, L., et al. (2008). Structure of the DNA repair helicase XPD. Cell 133, 801-812. doi: 10.1016/j.cell.2008.04.029

Liu, W., Zhou, M., Li, Z., Li, H., Polaczek, P., Dai, H., et al. (2016). A selective small molecule DNA2 inhibitor for sensitization of human cancer cells to chemotherapy. EBioMed. 6, 73-86. doi: 10.1016/j.ebiom.2016.02.043

Lu, L., Jin, W., and Wang, L. L. (2017). Aging in Rothmund-Thomson syndrome and related RECQL4 genetic disorders. Ageing Res. Rev. 33, 30-35. doi: 10.1016/j.arr.2016.06.002

Lucic, B., Zhang, Y., King, O., Mendoza-Maldonado, R., Berti, M., Niesen, F. H., et al. (2011). A prominent beta-hairpin structure in the winged-helix domain of RECQ1 is required for DNA unwinding and oligomer formation. Nucleic Acids Res. 39, 1703-1717. doi: 10.1093/nar/gkq1031

Macris, M. A., Krejci, L., Bussen, W., Shimamoto, A., and Sung, P. (2006). Biochemical characterization of the RECQ4 protein, mutated in Rothmund-Thomson syndrome. DNA Repair 5, 172-180. doi: 10.1016/j.dnarep.2005.09.005

Maga, G., Falchi, F., Garbelli, A., Belfiore, A., Witvrouw, M., Manetti, F., et al. (2008). Pharmacophore modeling and molecular docking led to the discovery of inhibitors of human immunodeficiency virus-1 replication targeting the human cellular aspartic acid-glutamic acid-alanine-aspartic acid box polypeptide 3. J. Med. Chem. 51, 6635-6638. doi: 10.1021/jm8008844

Mastrangelo, E., Pezzullo, M., De Burghgraeve, T., Kaptein, S., Pastorino, B., Dallmeier, K., et al. (2012). Ivermectin is a potent inhibitor of flavivirus replication specifically targeting NS3 helicase activity: new prospects for an old drug. J. Antimicrob. Chemother. 67, 1884-1894. doi: 10.1093/jac/dks147

Mazina, O. M., Rossi, M. J., Deakyne, J. S., Huang, F., and Mazin, A. V. (2012). Polarity and bypass of DNA heterology during branch migration of Holliday junctions by human RAD54, BLM, and RECQ1 proteins. J. Biol. Chem. 287, 11820-11832. doi: 10.1074/jbc.M112.341347

Meng, X. Y., Zhang, H. X., Mezei, M., and Cui, M. (2011). Molecular docking: a powerful approach for structure-based drug discovery. Curr. Comput. Aided Drug Des. 7, 146-157. doi: 10.2174/157340911795677602

Moitessier, N., Englebienne, P., Lee, D., Lawandi, J., and Corbeil, C. R. (2008). Towards the development of universal, fast and highly accurate docking/scoring methods: a long way to go. Br. J. Pharmacol. 153(Suppl. 1), S7-S26. doi: 10.1038/sj.bjp.0707515

Murata, S., Zhang, C., Finch, N., Zhang, K., Campo, L., and Breuer, E. K. (2016). Predictors and modulators of synthetic lethality: an update on PARP inhibitors and personalized medicine. Biomed Res. Int. 2016:2346585. doi: $10.1155 / 2016 / 2346585$

Neves, H., and Kwok, H. F. (2017). In sickness and in health: the many roles of the minichromosome maintenance proteins. Biochim. Biophys. Acta 1868, 295-308. doi: 10.1016/j.bbcan.2017.06.001

Newman, J. A., Aitkenhead, H., Savitsky, P., and Gileadi, O. (2017). Insights into the RecQ helicase mechanism revealed by the structure of the 
helicase domain of human RECQL5. Nucleic Acids Res. 45, 4231-4243. doi: $10.1093 / \mathrm{nar} / \mathrm{gkw} 1362$

Newman, J. A., Savitsky, P., Allerston, C. K., Bizard, A. H., Ozer, O., Sarlos, K., et al. (2015). Crystal structure of the Bloom's syndrome helicase indicates a role for the HRDC domain in conformational changes. Nucleic Acids Res. 43, 5221-5235. doi: 10.1093/nar/gkv373

Nguyen, G. H., Dexheimer, T. S., Rosenthal, A. S., Chu, W. K., Singh, D. K., Mosedale, G., et al. (2013). A small molecule inhibitor of the BLM helicase modulates chromosome stability in human cells. Chem. Biol. 20, 55-62. doi: 10.1016/j.chembiol.2012.10.016

O'Connor, M. J. (2015). Targeting the DNA damage response in cancer. Mol. Cell 60, 547-560. doi: 10.1016/j.molcel.2015.10.040

Oshima, J., Sidorova, J. M., and Monnat, R. J. Jr. (2017). Werner syndrome: clinical features, pathogenesis and potential therapeutic interventions. Ageing Res. Rev. 33, 105-114. doi: 10.1016/j.arr.2016.03.002

Pike, A. C., Gomathinayagam, S., Swuec, P., Berti, M., Zhang, Y., Schnecke, C., et al. (2015). Human RECQ1 helicase-driven DNA unwinding, annealing, and branch migration: insights from DNA complex structures. Proc. Natl. Acad. Sci. U.S.A. 112, 4286-4291. doi: 10.1073/pnas.1417594112

Pike, A. C., Shrestha, B., Popuri, V., Burgess-Brown, N., Muzzolini, L., Costantini, S., et al. (2009). Structure of the human RECQ1 helicase reveals a putative strand-separation pin. Proc. Natl. Acad. Sci. U.S.A. 106, 1039-1044. doi: 10.1073/pnas.0806908106

Pinter, S. F., Aubert, S. D., and Zakian, V. A. (2008). The Schizosaccharomyces pombe Pfhlp DNA helicase is essential for the maintenance of nuclear and mitochondrial DNA. Mol. Cell. Biol. 28, 6594-6608. doi: 10.1128/mcb.00191-08

Pommier, Y. (2013). Drugging topoisomerases: lessons and challenges. ACS Chem. Biol. 8, 82-95. doi: 10.1021/cb300648v

Pommier, Y., and Cherfils, J. (2005). Interfacial inhibition of macromolecular interactions: nature's paradigm for drug discovery. Trends Pharmacol. Sci. 26, 138-145. doi: 10.1016/j.tips.2005.01.008

Pommier, Y., Kiselev, E., and Marchand, C. (2015). Interfacial inhibitors. Bioorg. Med. Chem. Lett. 25, 3961-3965. doi: 10.1016/j.bmcl.2015.07.032

Pommier, Y., Leo, E., Zhang, H., and Marchand, C. (2010). DNA topoisomerases and their poisoning by anticancer and antibacterial drugs. Chem. Biol. 17, 421-433. doi: 10.1016/j.chembiol.2010.04.012

Pommier, Y., O'Connor, M. J., and de Bono, J. (2016). Laying a trap to kill cancer cells: PARP inhibitors and their mechanisms of action. Sci. Transl. Med. 8:362ps317. doi: $10.1126 /$ scitranslmed.aaf 9246

Pugh, R. A., Honda, M., Leesley, H., Thomas, A., Lin, Y., Nilges, M. J., et al. (2008). The iron-containing domain is essential in Rad3 helicases for coupling of ATP hydrolysis to DNA translocation and for targeting the helicase to the singlestranded DNA-double-stranded DNA junction. J. Biol. Chem. 283, 1732-1743. doi: 10.1074/jbc.M707064200

Pugh, R. A., Wu, C. G., and Spies, M. (2012). Regulation of translocation polarity by helicase domain 1 in SF2B helicases. EMBO J. 31, 503-514. doi: 10.1038/emboj.2011.412

Radi, M., Falchi, F., Garbelli, A., Samuele, A., Bernardo, V., Paolucci, S., et al. (2012). Discovery of the first small molecule inhibitor of human DDX3 specifically designed to target the RNA binding site: towards the next generation HIV-1 inhibitors. Bioorg. Med. Chem. Lett. 22, 2094-2098. doi: 10.1016/j.bmcl.2011.12.135

Rosenthal, A. S., Dexheimer, T. S., Gileadi, O., Nguyen, G. H., Chu, W. K., Hickson, I. D., et al. (2013). Synthesis and SAR studies of 5-(pyridin4-yl)-1,3,4-thiadiazol-2-amine derivatives as potent inhibitors of Bloom helicase. Bioorg. Med. Chem. Lett. 23, 5660-5666. doi: 10.1016/j.bmcl.2013. 08.025

Rudolf, J., Makrantoni, V., Ingledew, W. J., Stark, M. J., and White, M. F. (2006). The DNA repair helicases XPD and FancJ have essential iron-sulfur domains. Mol. Cell 23, 801-808. doi: 10.1016/j.molcel.2006.07.019

Shadrick, W. R., Ndjomou, J., Kolli, R., Mukherjee, S., Hanson, A. M., and Frick, D. N. (2013). Discovering new medicines targeting helicases: challenges and recent progress. J. Biomol. Screen. 18, 761-781. doi: 10.1177/1087057113482586

Shamanna, R. A., Lu, H., Croteau, D. L., Arora, A., Agarwal, D., Ball, G., et al. (2016). Camptothecin targets WRN protein: mechanism and relevance in clinical breast cancer. Oncotarget 7, 13269-13284. doi: 10.18632/oncotarget.7906
Shoichet, B. K., McGovern, S. L., Wei, B., and Irwin, J. J. (2002). Lead discovery using molecular docking. Curr. Opin. Chem. Biol. 6, 439-446. doi: 10.1016/S1367-5931(02)00339-3

Simon, N., Bochman, M. L., Seguin, S., Brodsky, J. L., Seibel, W. L., and Schwacha, A. (2013). Ciprofloxacin is an inhibitor of the Mcm2-7 replicative helicase. Biosci. Rep. 33:e00072. doi: 10.1042/bsr20130083

Suhasini, A. N., and Brosh, R. M. Jr. (2013). Disease-causing missense mutations in human DNA helicase disorders. Mutat. Res. 752, 138-152. doi: 10.1016/j.mrrev.2012.12.004

Symington, L. S. (2016). Mechanism and regulation of DNA end resection in eukaryotes. Crit. Rev. Biochem. Mol. Biol. 51, 195-212. doi: 10.3109/10409238.2016.1172552

Teuscher, K. B., Zhang, M., and Ji, H. (2017). A versatile method to determine the cellular bioavailability of small-molecule inhibitors. J. Med. Chem. 60, 157-169. doi: 10.1021/acs.jmedchem.6b00923

Thangavel, S., Berti, M., Levikova, M., Pinto, C., Gomathinayagam, S., Vujanovic, M., et al. (2015). DNA2 drives processing and restart of reversed replication forks in human cells. J. Cell Biol. 208, 545-562. doi: 10.1083/jcb.201406100

Toyokawa, G., Masuda, K., Daigo, Y., Cho, H. S., Yoshimatsu, M., Takawa, M., et al. (2011). Minichromosome Maintenance Protein 7 is a potential therapeutic target in human cancer and a novel prognostic marker of non-small cell lung cancer. Mol. Cancer 10:65. doi: 10.1186/1476-4598-10-65

Tripathi, A., and Bankaitis, V. A. (2017). Molecular docking: from lock and key to combination lock. J. Mol. Med. Clin. Appl. 2. doi: 10.16966/2575-0305.106

Tyring, S., Wald, A., Zadeikis, N., Dhadda, S., Takenouchi, K., and Rorig, R. (2012). ASP2151 for the treatment of genital herpes: a randomized, doubleblind, placebo- and valacyclovir-controlled, dose-finding study. J. Infect. Dis. 205, 1100-1110. doi: 10.1093/infdis/jis019

Umate, P., Tuteja, N., and Tuteja, R. (2011). Genome-wide comprehensive analysis of human helicases. Commun. Integr. Biol. 4, 118-137. doi: 10.4161/cib.4.1.13844

Velic, D., Couturier, A. M., Ferreira, M. T., Rodrigue, A., Poirier, G. G., Fleury, F., et al. (2015). DNA damage signalling and repair inhibitors: the long-sought-after achilles' heel of cancer. Biomolecules 5, 3204-3259. doi: 10.3390/biom5043204

White, P. W., Titolo, S., Brault, K., Thauvette, L., Pelletier, A., Welchner, E., et al. (2003). Inhibition of human papillomavirus DNA replication by small molecule antagonists of the E1-E2 protein interaction. J. Biol. Chem. 278, 26765-26772. doi: $10.1074 /$ jbc.M303608200

Wolski, S. C., Kuper, J., Hanzelmann, P., Truglio, J. J., Croteau, D. L., Van Houten, B., et al. (2008). Crystal structure of the FeS clustercontaining nucleotide excision repair helicase XPD. PLoS Biol. 6:e149. doi: 10.1371/journal.pbio.0060149

Workman, P., and Collins, I. (2010). Probing the probes: fitness factors for small molecule tools. Chem. Biol. 17, 561-577. doi: 10.1016/j.chembiol.2010.05.013

Wu, L., Chan, K. L., Ralf, C., Bernstein, D. A., Garcia, P. L., Bohr, V. A., et al. (2005). The HRDC domain of BLM is required for the dissolution of double Holliday junctions. EMBO J. 24, 2679-2687. doi: 10.1038/sj.emboj.7600740

$\mathrm{Wu}, \mathrm{Y}$., and Brosh, R. M. Jr. (2012). DNA helicase and helicase-nuclease enzymes with a conserved iron-sulfur cluster. Nucleic Acids Res. 40, 4247-4260. doi: $10.1093 / \mathrm{nar} / \mathrm{gks} 039$

Wu, Y., Sommers, J. A., Suhasini, A. N., Leonard, T., Deakyne, J. S., Mazin, A. V., et al. (2010). Fanconi anemia group J mutation abolishes its DNA repair function by uncoupling DNA translocation from helicase activity or disruption of protein-DNA complexes. Blood 116, 3780-3791. doi: 10.1182/blood-2009-11-256016

$\mathrm{Xu}, \mathrm{X}$., and Liu, Y. (2009). Dual DNA unwinding activities of the Rothmund-Thomson syndrome protein, RECQ4. EMBO J. 28, 568-577. doi: $10.1038 /$ emboj.2009.13

Yedavalli, V. S., Zhang, N., Cai, H., Zhang, P., Starost, M. F., Hosmane, R. S., et al. (2008). Ring expanded nucleoside analogues inhibit RNA helicase and intracellular human immunodeficiency virus type 1 replication. J. Med. Chem. 51, 5043-5051. doi: $10.1021 / \mathrm{jm} 800332 \mathrm{~m}$

Yoakim, C., Ogilvie, W. W., Goudreau, N., Naud, J., Hache, B., O’Meara, J. A., et al. (2003). Discovery of the first series of inhibitors of human papillomavirus type 11: inhibition of the assembly of the E1-E2-Origin DNA complex. Bioorg. Med. Chem. Lett. 13, 2539-2541. doi: 10.1016/S0960-894X(03)00510-9 
Zahreddine, H., and Borden, K. L. (2013). Mechanisms and insights into drug resistance in cancer. Front. Pharmacol. 4:28. doi: 10.3389/fphar.2013. 00028

Zhai, Y., and Tye, B. K. (2017). Structure of the MCM2-7 Double Hexamer and Its Implications for the Mechanistic Functions of the Mcm2-7 Complex. Adv. Exp. Med. Biol. 1042, 189-205. doi: 10.1007/978-981-10-6 955-0_9

Zhou, C., Pourmal, S., and Pavletich, N. P. (2015). Dna2 nucleasehelicase structure, mechanism and regulation by Rpa. Elife 4:e09832. doi: $10.7554 /$ eLife. 09832
Conflict of Interest Statement: The authors declare that the research was conducted in the absence of any commercial or financial relationships that could be construed as a potential conflict of interest.

Copyright (c) 2018 Datta and Brosh. This is an open-access article distributed under the terms of the Creative Commons Attribution License (CC BY). The use, distribution or reproduction in other forums is permitted, provided the original author(s) and the copyright owner are credited and that the original publication in this journal is cited, in accordance with accepted academic practice. No use, distribution or reproduction is permitted which does not comply with these terms. 